\title{
PRIMARY CHARACTERISTIC HOMOMORPHISM OF PAIRS OF LIE ALGEBROIDS AND MACKENZIE ALGEBROID
}

\author{
BOGDAN BALCERZAK ${ }^{1}$ \\ JAN KUBARSKI ${ }^{1,2}$ \\ WITOLD WALAS ${ }^{1}$ \\ ${ }^{1}$ Institute of Mathematics, Technical University of Eódź \\ al. Politechniki 11, 90-924 Eódź, Poland \\ E-mail:bogdan@ck-sg.p.lodz.pl,kubarski@ck-sg.p.lodz.pl,walwit@ck-sg.p.lodz.pl \\ ${ }^{2}$ Institute of Mathematics and Informatics \\ Częstochowa Technical University \\ Dabrowskiego 69, 42-201 Częstochowa, Poland
}

\begin{abstract}
Connections for a pair of Lie algebroids are defined as linear homomorphisms between these Lie algebroids, commuting with the anchors. The primary characteristic homomorphism of a pair of Lie algebroids is defined and compared with other known Chern-Weil homomorphisms.
\end{abstract}

1. Introduction. By an $L$-connection in $A$ ( $L$ and $A$ are Lie algebroids on the same manifold $B$ ) we mean a linear homomorphism $\nabla: L \rightarrow A$ commuting with the anchors $\omega_{A} \circ \nabla=\omega_{L}$. This definition covers

- usual and partial covariant derivatives in vector bundles,

- usual and partial connections in principal bundles,

- connections in transitive or regular Lie algebroids,

— connections in extensions e : $0 \rightarrow L^{\prime} \rightarrow A \rightarrow L \rightarrow 0$ of Lie algebroids, in particular, complete differentials of higher order,

- transversal connections in extensions of principal fibre bundles,

- covariant and contravariant connections known in Poisson geometry.

2000 Mathematics Subject Classification: Primary 57R20; Secondary 53C05.

Key words and phrases: Lie algebroid, extension of Lie algebroids, connection, partial connection, transversal connection, covariant derivative, contravariant connection, Chern-Weil homomorphism.

The paper is in final form and no version of it will be published elsewhere. 
$L$-connections in vector bundles and $L$-connections in principal bundles [F2] determine $L$-connections in suitable Lie algebroids of vector bundles and principal bundles.

In this work we construct the Chern-Weil homomorphism $h_{L, A}$ of a pair $(L, A)$ of Lie algebroids, where $A$ is regular, giving obstructions to the existence of flat $L$-connections in A. This approach generalizes known constructions by Teleman 1972 [T2], Mackenzie 1988 [M5], Kubarski 1991 [K4], Vaisman 1994 [V2], Belko 1997 [B2], Huebschmann 1999 [H2], Itskov, Karasev and Vorobjev 1999 [IKV], Fernandes (2000) [F1], [F2], Crainic (preprint 2001) [C]. For the tangential case see also Moore and Schochet 1988 [M-C], Kubarski $1993[\mathrm{~K} 5]$.

Next, $h_{L, A}$ is compared with the other Chern-Weil homomorphisms $h_{L}, h_{A}$, and $h_{\mathbf{e}}$ in the case of an extension e. We also introduce a $G$-invariant Chern-Weil homomorphism for $G$-algebroids and use it to transversal $P B G$-Mackenzie algebroids.

(1) A covariant derivative in a vector bundle $\mathfrak{f}$ on a smooth manifold $B$ is a mapping $\nabla: \mathfrak{X}(B) \rightarrow \operatorname{End}_{\mathbb{R}}(\operatorname{Sec} \mathfrak{f})$ which satisfies the known Koszul axioms $[\mathrm{K}]: \nabla_{X} \sigma$ is $C^{\infty}(B)$ linear with respect to $X \in \mathfrak{X}(B)$ and $\mathbb{R}$-linear with respect to $\sigma \in \operatorname{Sec} \mathfrak{f}$, while $\nabla_{X}$ : $\operatorname{Sec} \mathfrak{f} \rightarrow \operatorname{Sec} \mathfrak{f}$ is a covariant differential operator with the anchor $X$ (i.e. $\nabla_{X}(f \cdot \sigma)=$ $\left.f \cdot \nabla_{X} \sigma+X(f) \cdot \sigma\right)$. Cohomology classes from the image of the primary characteristic homomorphism of $\mathfrak{f}$ determine topological obstructions to existence of a flat covariant derivative in $\mathfrak{f}$.

If we replace $\mathfrak{X}(B)$ with $\operatorname{Sec} F$ (where $F \subset T B$ is a vector subbundle tangent to some regular foliation on $B$ ), then we obtain the so-called partial covariant derivative in the bundle $\mathfrak{f}[\mathrm{K}-\mathrm{T}]$. In both cases, $T B$ and $F$ are trivial Lie algebroids, whereas the space of covariant differential operators in the bundle $\mathfrak{f}$ is the space of cross-sections of the Lie algebroid $A(\mathfrak{f})$ of $\mathfrak{f}$, see [K4], [K8], [M1], [K3], denoted also by $C D O(\mathfrak{f})$. An operator $\nabla$ can be given equivalently as a linear homomorphism of vector bundles $\nabla: T B \rightarrow A(\mathfrak{f})$ $[\nabla: F \rightarrow A(\mathfrak{f})]$ such that $\omega_{\mathfrak{f}} \circ \nabla=\mathrm{id}$, where $\omega_{\mathfrak{f}}: A(\mathfrak{f}) \rightarrow T B$ is the anchor of the Lie algebroid $A(\mathfrak{f})$. The next one, very important in differential geometry generalizations, can be obtained by taking any Lie algebroid $\left(L, \llbracket \cdot, \cdot \rrbracket, \omega_{L}\right)$ instead of $T B$,

$$
\nabla: L \rightarrow A(\mathfrak{f})
$$

assuming additionally that $\nabla$ commutes with anchors

$$
\omega_{\mathfrak{f}} \circ \nabla=\omega_{L} .
$$

Then the operator $\nabla$ is said to be an $L$-covariant derivative in the vector bundle $\mathfrak{f}$ (cf. the definition of an $L$-connection in the more general category of Lie-Rinehart algebras [H1], [H2]).

The operator (1.1) induces the so-called linear $L$-connection in $\mathfrak{f}, \nabla: \operatorname{Sec} L \times \operatorname{Sec} \mathfrak{f} \rightarrow$ Sec $\mathfrak{f}$ (introduced in the case of Poisson manifolds $B$ by I. Vaisman [V1] for $L=T^{*} B$ and known as a contravariant derivative), see also [H-M].

In the case when the operator (1.1) is a homomorphism of Lie algebroids it is known as a representation of $L$ in $\mathfrak{f}$. Further, if we replace $A(\mathfrak{f})$ with a Lie algebroid $\left(A, \llbracket \cdot, \cdot \rrbracket, \omega_{A}\right)$, then we obtain a quite general object (examined in this work)

$$
\nabla: L \rightarrow A, \omega_{A} \circ \nabla=\omega_{L},
$$


called an $L$-connection in the Lie algebroid $A$. In the case when $A=T P_{/ G}$ is the Lie algebroid of a principal bundle $P(B, G, p)$ and $L=T B$, considered connections correspond to connections in the principal bundle $P$ (for $L \subset T B$-partial connections). If $A=T P_{/ G}$ (as above) and $L=T^{*} B$ is the Lie algebroid of a Poisson manifold $B$, then any operator (1.1) is determined by the so-called contravariant connection $h: p^{*} T^{*} B \rightarrow T P$ (introduced recently by R. L. Fernandes [F1]) and vice versa.

If $A$ is a transitive Lie algebroid and $L$ is an arbitrary one, both over the manifold $B$, and the characteristic Stefan foliation $\operatorname{Im} \omega_{L}$ is contained in a regular foliation $F \subset T B$, $\operatorname{Im} \omega_{L} \subset F$, then any $L$-connection $\nabla: L \rightarrow A$ has values in the regular Lie algebroid $A^{F}=\omega_{A}^{-1}[F]$ so $\nabla$ determines an $L$-connection in $A^{F}, \nabla^{F}: L \rightarrow A^{F}, \nabla^{F}(v)=\nabla(v)$. We should add here that the domain $I\left(A^{F}\right)$ of the Chern-Weil homomorphism $h_{A^{F}}$ of $A^{F}$ [K4], [K5] contains the $\Omega_{b}(B, F)$-module $\Omega_{b}(B, F) \cdot I(A)$ (i.e. linear combinations $\left.\sum f^{i} \cdot \Gamma_{i}, f^{i} \in \Omega_{b}(B, F), \Gamma_{i} \in I(A)\right)$, where $I(A)$ is the domain of $h_{A}$ and $\Omega_{b}(B, F)$ is the space of $F$-basic functions. Sometimes (and this is rather interesting), $I\left(A^{F}\right)$ is larger than $\Omega_{b}(B, F) \cdot I(A)[K 5]$ so it may be a source of new "singular" characteristic classes which are obstructions to the existence of flat $L$-connections in $A$, see below.

(2) In this paper we construct a characteristic homomorphism $h_{L, A}: I(A) \rightarrow H_{L}(B)$ of the Chern-Weil type, which describes obstructions to the existence of flat $L$-connections $\nabla$ in a regular Lie algebroid $A$, where $I(A)$ is the algebra of real multilinear symmetric homomorphisms of the adjoint bundle of Lie algebras $\boldsymbol{g}_{A}=\operatorname{ker} \omega_{A}$ and invariant with respect to the adjoint representation of $A$ on $\boldsymbol{g}_{A}$ while $H_{L}(B)$ is a cohomology algebra of real $A$-forms [MR], [M1], [K4]. The domain of $h_{L, A}$ is equal to the domain of $h_{A}$. We have the equalities $h_{T B, A}=h_{A}$ and $h_{F, A}=h_{A^{F}}$ (for a regular foliation $F \subset T B$ ). The relation between $h_{A}$ and $h_{L, A}$ is $\omega_{L}^{\#} \circ h_{A}=h_{L, A}$. Putting $L=A$ we obtain $\omega_{A}^{\sharp} \circ h_{A}=h_{A, A}=0$ which gives easily that $\operatorname{Pont}(A)=\operatorname{Im} h_{A} \subset \operatorname{ker} \omega_{A}^{\sharp}$.

The homomorphism $h_{L, A}$ is a generalization of:

1. the classical Chern-Weil homomorphism of a vector bundle $\mathfrak{f}$ (if $A=A(\mathfrak{f}$ ) and $L=T B)$,

2. the tangential Chern-Weil homomorphism of $\mathfrak{f}$ over a foliated manifold [M-C], [K5] (if $A=A(\mathfrak{f})$ and $L=F \subset T B$ ),

3. the characteristic homomorphism of regular Lie algebroid [K4] (if $L=F=\operatorname{Im}\left(\omega_{A}\right)$; in this case an $F$-connection in $A$ is a splitting $\nabla: F \rightarrow A$ of the Atiyah sequence in $\left.A, 0 \rightarrow \boldsymbol{g}_{A} \rightarrow A \rightarrow F \rightarrow 0\right)$,

4. the Chern-Weil homomorphism of a principal bundle, if $A=A(P)=T P_{/ G}$ and $L=T B$,

5. the Fernandes [F2] and Crainic [C] $L$-Chern-Weil homomorphism of a real vector bundle $\mathfrak{f}$ (if $A=A(\mathfrak{f})$ ).

A comparison of $h_{L, A(P)}$ to the Fernandes $L$-Chern-Weil homomorphism of a principal bundle $P[\mathrm{~F} 2]$ is given in section 3.5 below.

(3) Recall (cf. (4) below) [K4], [B1], [B2] the relation between the classical Chern-Weil homomorphism $h_{P}$ of a $G$-principal fibre bundle $P(B, G)$ and the Chern-Weil homomorphism $h_{A(P)}$ of the transitive Lie algebroid $A(P)=T P_{/ G}$ of $P$ : there exists a monomorphism of algebras $\nu: I(G) \rightarrow I(A(P))$ which gives commutativity of the diagram 


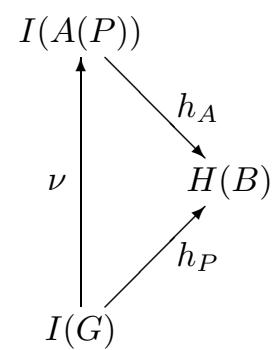

If the total space of $P$ is a connected manifold (the structure Lie group need not be connected), then $\nu$ is an isomorphism [K4], [B1], [B2]. Notice that if the base manifold $B$ is connected, then the connectedness of $P$ is equivalent to the connectedness of the Ehresmann groupoid $P \times_{G} P$ [Lis].

Recently R. L. Fernandes [F2] has obtained an $L$-Chern-Weil homomorphism of a $G$-principal fibre bundle $P$ which turns out to be equal to $h_{L, A(P)}$ if the domains of $h_{P}$ and $h_{A(P)}$ are the same (for example, if $P$ is connected). The Fernandes case is very important in Poisson geometry.

(4) V. Itskov, M. Karasev and Yu. Vorobjev [IKV] had constructed independently a Chern-Weil homomorphism for a transitive Lie algebroid $\left(A \rightarrow B, \omega_{A},\{\},\right)$. It is a homomorphism

$$
h: \bigoplus^{k} \operatorname{Inv}^{k}\left(\boldsymbol{g}_{\mid x_{0}}\right) \rightarrow H_{d R}(B), \Gamma \mapsto[\Gamma(R, \ldots, R)],
$$

where $R$ is the curvature of any connection in $A$ and $\boldsymbol{g}_{\mid x_{0}}$ is the isotropy Lie algebra of $A$ at $x_{0}$, i.e. the fibre of $\boldsymbol{g}$ at $x_{0} \in B$. The domain $\bigoplus^{k} \operatorname{Inv}^{k}\left(\boldsymbol{g}_{\mid x_{0}}\right)$ is in general smaller than the domain $I(A)$ considered in the previous papers [T2], [K4], [B2], [H2], however sometimes equality holds. The domain $I(A)=\bigoplus^{k} \operatorname{Sec}\left(\bigvee^{k} \boldsymbol{g}^{*}\right)_{I(\mathrm{ad})}$ is the algebra of ad-invariant cross-sections of the symmetric powers $\bigvee^{k} \boldsymbol{g}^{*}$. Equivalently, it is the algebra of parallel sections - under the flat adjoint connection - of the vector bundles $\operatorname{inv}^{k}(\boldsymbol{g})$ whose fibres are the spaces of ad-invariant polynomials on $\boldsymbol{g}_{\mid x}$, which can be described by K. Mackenzie's formula in Th. IV.1.19 of [M1]. This formula says that an element from inv ${ }^{k}\left(\boldsymbol{g}_{\mid x_{0}}\right)$ can be extended to a parallel cross-section if and only if it is invariant with respect to the $\pi_{1}(B)$-action on $\operatorname{inv}^{k}\left(\boldsymbol{g}_{\mid x_{0}}\right)$ via the holonomy morphism for any flat adjoint connection on the vector bundle $\operatorname{inv}^{k}(\boldsymbol{g})$. To better understand the relation between the domains $\bigoplus^{k} \operatorname{Inv}^{k}\left(\boldsymbol{g}_{\mid x_{0}}\right)$ and $I(A)$ notice first that the space $\operatorname{Inv}^{k}\left(\boldsymbol{g}_{\mid x_{0}}\right)$ after naturally extending its elements to parallel cross-sections of $\operatorname{inv}^{k}(\boldsymbol{g})$, is equal to the space of invariant elements with respect to the canonical representation $T$ of the $\operatorname{Aut}\left(\boldsymbol{g}_{\mid x_{0}}\right)$-principal bundle $\operatorname{Aut}(\boldsymbol{g})$ of Lie algebra isomorphisms $\boldsymbol{g}_{\mid x_{0}} \stackrel{\cong}{\longrightarrow} \boldsymbol{g}_{\mid x}$ on the vector bundle $\bigvee^{k} \boldsymbol{g}^{*}$, induced by the inclusion $\operatorname{Aut}(\boldsymbol{g}) \subset L(\boldsymbol{g})\left[L(\boldsymbol{g})\right.$ is the full $\mathrm{GL}\left(\boldsymbol{g}_{\mid x_{0}}\right)$-principal bundle of frames of $\boldsymbol{g}$, and $\left.T(z)=\bigvee^{k}\left(z^{*}\right)^{-1}, z: \boldsymbol{g}_{\mid x_{0}} \rightarrow \boldsymbol{g}_{\mid x}\right]$. Next consider the sequence of inclusions

$$
\operatorname{Inv}^{k}\left(\boldsymbol{g}_{\mid x_{0}}\right) \subset \operatorname{Sec}\left(\bigvee^{k} \boldsymbol{g}^{*}\right)_{I(d)} \subset \operatorname{Sec}\left(\bigvee^{k} \boldsymbol{g}^{*}\right)_{I(\mathrm{ad})}
$$

where the middle element consists of all invariant cross-sections with respect to the derivative of $T$. According to Prop. 3.3.8 of [K5] this element is equal to the space of cross-sections $\Gamma \in \operatorname{Sec}\left(\bigvee^{k} \boldsymbol{g}^{*}\right)$ invariant with respect to all covariant differential operators 
$\xi: \operatorname{Sec}(\boldsymbol{g}) \rightarrow \operatorname{Sec}(\boldsymbol{g})$ which are differentiations of the Lie algebra $\operatorname{Sec}(\boldsymbol{g})$, i.e. cross-sections $\Gamma$ such that

$$
\left(\omega_{A} \circ \xi\right)\left(\Gamma\left(\theta_{1}, \ldots, \theta_{k}\right)\right)-\sum_{i} \Gamma\left(\theta_{1}, \ldots, \xi\left(\theta_{i}\right), \ldots, \theta_{k}\right)=0
$$

for all $\xi$ as above. Since there are, in general, fewer adjoint differentiations than all differentiations, it can give sometimes the effect that the second inclusion is not an equality. According to Prop. 5.5.3 of [K4] the first inclusion is an equation if the $\operatorname{Aut}\left(\boldsymbol{g}_{\mid x_{0}}\right)$-principal bundle $\operatorname{Aut}(\boldsymbol{g})$ is connected.

(5) In the present paper we compare the constructed Chern-Weil homomorphism of pairs of Lie algebroids with the other one given by J. Huebschmann [H2] for splittings of Lie-Rinehart algebras (and earlier, in a less general case, by N. Teleman [T1], [T2]), which in the case of Lie algebroids relates to the epimorphism of Lie algebroids $A \stackrel{\pi}{\rightarrow} L$ and connection interpreted as its right inverse in the class of linear homomorphisms $\nabla: L \rightarrow A, \pi \circ \nabla=$ id. Diagram (3.8) in part 3 below compares four Chern-Weil homomorphisms $h_{A}, h_{L}, h_{L, A}, h_{\mathbf{e}}$ for a given extension e : $0 \rightarrow L^{\prime} \rightarrow A \stackrel{\pi}{\rightarrow} L \rightarrow 0$.

Complete differentials of higher order in the tangent bundle $T B[\mathrm{P}],[\mathrm{NVQ}],[\mathrm{S}]$, given as splittings of the exact sequence of the jet bundles $0 \rightarrow S^{k}(T B, T B) \rightarrow J^{k}(T B) \rightarrow$ $J^{k-1}(T B) \rightarrow 0$, are important examples of right inverse connections.

Other important examples are given by K. Mackenzie [M2] in connection with extensions of principal bundles. Commutativity of diagram (3.8) on the level of transitive Lie algebroids and the epimorphism $\pi: A \rightarrow L$ given by the extension of principal bundles $Q(B, H, q) \rightarrow P(B, G, p)$ (with respect to the extension of Lie groups $0 \rightarrow N \rightarrow H \rightarrow G \rightarrow 0$ ) - with $h_{L, A}$ omitted - is described in [M2, Prop. 3.7]. It is done in terms of the Chern-Weil homomorphisms of principal bundles $P(B, G), Q(B, H)$ and the transversal Chern-Weil homomorphism of the transversal principal bundle $Q(P, N)$, and expressed as commutativity of the following diagram

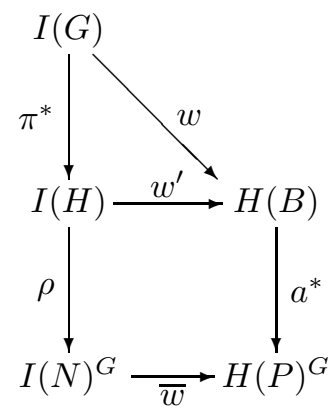

( $a$ denotes the anchor of $P(B, G)$; $\bar{w}$ is equivalent to the Chern-Weil homomorphism of the extension of transitive Lie algebroids $\left.0 \rightarrow Q \times_{H} \mathfrak{n} \rightarrow T Q_{/ H} \rightarrow T P_{/ G} \rightarrow 0\right)$.

(6) Next, the $G$-equivariant Chern-Weil homomorphism for an extension of $G$-algebroids is introduced (the notion of a $G$-algebroid comes from [K6] and generalizes the notion of a Mackenzie $P B G$-algebroid [M4]).

Finally we examine extension (2.3) in which $L$ is an integrable Lie algebroid $L=$ $T P / G$. According to [M5] it produces a transversal $P B G$-Mackenzie algebroid $p^{*} A$ (where 
$p: P \rightarrow B$ is the projection). An equivalence of the Chern-Weil homomorphism $h_{\mathbf{e}}$ of $\mathbf{e}$ $: 0 \rightarrow L^{\prime} \rightarrow A \stackrel{\pi}{\rightarrow} T P_{/ G} \rightarrow 0$ and the $G$-equivariant Chern-Weil homomorphism $h_{p^{*} A}^{G}$ of the $G$-algebroid $p^{*} A$ is obtained which generalizes the Mackenzie result concerning extension $Q(B, H, q) \stackrel{\pi}{\rightarrow} P(B, G, p) \rightarrow 0$ of principal bundles.

Some of these results were presented in the poster $[\mathrm{K}-\mathrm{W}]$ during the 3rd ECM, Barcelona 2000.

\section{L-connections}

2.1. Definitions and examples. A Lie algebroid $[\mathrm{Pr}]$ on a manifold $B$ is a triple $\left(L, \llbracket \cdot, \cdot \rrbracket, \omega_{L}\right)$ where $L$ is a vector bundle on $B,(\operatorname{Sec} L, \llbracket \cdot, \cdot \rrbracket)$ is an $\mathbb{R}$-Lie algebra, $\omega_{L}: L \rightarrow$ $T B$ is a linear homomorphism of vector bundles and the following Leibniz condition is satisfied

$$
\llbracket \xi, f \cdot \eta \rrbracket=f \cdot \llbracket \xi, \eta \rrbracket+\omega_{L}(\xi)(f) \cdot \eta, \quad f \in C^{\infty}(B), \xi, \eta \in \operatorname{Sec} L .
$$

Lemma 2.1 (See $[\mathrm{H}])$. The anchor is bracket-preserving, $\omega_{L} \llbracket \xi, \eta \rrbracket=\left[\omega_{L} \xi, \omega_{L} \eta\right]$.

Proof. Let $\xi, \eta, \tau \in \operatorname{Sec} L, f \in C^{\infty}(B)$. Observe that

$$
\begin{aligned}
\left(\omega_{L} \circ \xi\right)\left(\left(\omega_{L} \circ \eta\right)(f)\right) \cdot \tau & =\llbracket \xi,\left(\left(\omega_{L} \circ \eta\right)(f)\right) \cdot \tau \rrbracket-\left(\left(\omega_{L} \circ \eta\right)(f)\right) \cdot \llbracket \xi, \tau \rrbracket \\
& =\llbracket \xi, \llbracket \eta, f \cdot \tau \rrbracket-f \cdot \llbracket \eta, \tau \rrbracket \rrbracket-(\llbracket \eta, f \cdot \llbracket \xi, \tau \rrbracket \rrbracket-f \cdot \llbracket \eta, \llbracket \xi, \tau \rrbracket \rrbracket) \\
& =\llbracket \xi, \llbracket \eta, f \cdot \tau \rrbracket \rrbracket-\llbracket \xi, f \cdot \llbracket \eta, \tau \rrbracket \rrbracket-\llbracket \eta, f \cdot \llbracket \xi, \tau \rrbracket \rrbracket+f \cdot \llbracket \eta, \llbracket \xi, \tau \rrbracket \rrbracket,
\end{aligned}
$$

and analogously

$$
\left(\omega_{L} \circ \eta\right)\left(\left(\omega_{L} \circ \xi\right)(f)\right)=\llbracket \eta, \llbracket \xi, f \cdot \tau \rrbracket \rrbracket-\llbracket \eta, f \cdot \llbracket \xi, \tau \rrbracket \rrbracket-\llbracket \xi, f \cdot \llbracket \eta, \tau \rrbracket \rrbracket+f \cdot \llbracket \xi, \llbracket \eta, \tau \rrbracket \rrbracket .
$$

Then

$$
\begin{aligned}
{\left[\omega_{L} \xi, \omega_{L} \eta\right](f) \cdot \tau=} & \left(\omega_{L} \circ \xi\right)\left(\left(\omega_{L} \circ \eta\right)(f)\right)-\left(\omega_{L} \circ \eta\right)\left(\left(\omega_{L} \circ \xi\right)(f)\right) \\
= & \llbracket \xi, \llbracket \eta, f \cdot \tau \rrbracket \rrbracket-\llbracket \xi, f \cdot \llbracket \eta, \tau \rrbracket \rrbracket-\llbracket \eta, f \cdot \llbracket \xi, \tau \rrbracket \rrbracket+f \cdot \llbracket \eta, \llbracket \xi, \tau \rrbracket \rrbracket \\
& -\llbracket \eta, \llbracket \xi, f \cdot \tau \rrbracket \rrbracket+\llbracket \eta, f \cdot \llbracket \xi, \tau \rrbracket \rrbracket+\llbracket \xi, f \cdot \llbracket \eta, \tau \rrbracket \rrbracket-f \cdot \llbracket \xi, \llbracket \eta, \tau \rrbracket \rrbracket \\
= & \llbracket \xi, \llbracket \eta, f \cdot \tau \rrbracket \rrbracket+\llbracket \eta, \llbracket f \cdot \tau, \xi \rrbracket \rrbracket+f \cdot(\llbracket \eta, \llbracket \xi, \tau \rrbracket \rrbracket+\llbracket \xi, \llbracket \tau, \eta \rrbracket \rrbracket) \\
= & -\llbracket f \cdot \tau, \llbracket \xi, \eta \rrbracket \rrbracket-f \cdot \llbracket \tau, \llbracket \eta, \xi \rrbracket \rrbracket \\
= & \llbracket \llbracket \xi, \eta \rrbracket, f \cdot \tau \rrbracket-f \cdot \llbracket \llbracket \xi, \eta \rrbracket, \tau \rrbracket=\left(\omega_{L} \circ \llbracket \xi, \eta \rrbracket\right)(f) \cdot \tau . \square
\end{aligned}
$$

REMARK 2.2. The analogous fact for an $(R, A)$-Lie-Rinehart algebra $(L, \omega)$ holds provided that $A$-module $L$ fulfils the axiom

- The representation $\rho: A \rightarrow \operatorname{End}_{A}(L), \rho(f)(\tau)=f \cdot \tau$, is faithful, i.e. $\operatorname{ker} \rho=0$.

Nontrivial Lie algebroids appeared as infinitesimal objects for Lie grupoids, principal bundles, vector bundles, TC-foliations, Poisson or Jacobi manifolds, etc. and play an analogous role to the Lie algebra of a Lie group (for review see [M3] and [K7]). If $\omega_{L}$ is of a constant rank, then a Lie algebroid is called regular, moreover, $\operatorname{Im} \omega_{L} \subset T B$ is an involutive distribution which determines a regular foliation (called also characteristic) on the base manifold $B$. Given any Lie algebroids on the same manifold the notion of a strong homomorphism is defined which preserves structures of Lie algebras and commutes with anchors, whereas in any case we have a less obvious notion of a nonstrong homomorphism, given by K. Mackenzie [H-M] (see also [K4]). 
Let $\left(L, \llbracket \cdot, \cdot \rrbracket, \omega_{L}\right)$ and $\left(A, \llbracket \cdot, \cdot \rrbracket, \omega_{A}\right)$ be Lie algebroids on a smooth manifold $B$ (we do not assume regularity of these algebroids).

Definition 2.3. A strong homomorphism of vector bundles $\nabla: L \rightarrow A$ is called an $L$-connection in $A$ if

$$
\omega_{A} \circ \nabla=\omega_{L}
$$

The existence of an $L$-connection in $A$ implies the inclusion of characteristic foliations $\operatorname{Im} \omega_{L} \subset \operatorname{Im} \omega_{A}$.

EXAMPLE 2.4. (1) Let $A$ be a regular Lie algebroid with the Atiyah sequence $0 \rightarrow$ $\boldsymbol{g}_{A} \rightarrow A \stackrel{\omega_{A}}{\rightarrow} F \rightarrow 0$. The splitting of this sequence $\nabla: F \rightarrow A, \omega \circ \nabla=\operatorname{id}_{F}$, is an $F$-connection in $A$. Clearly, $\omega_{A}$ is a unique $A$-connection in $F$. In [K4] we defined the Chern-Weil homomorphism of $A, h_{A}: I(A) \rightarrow H_{F}(B)$, where $I(A)$ is the space of invariant symmetric multilinear mappings on $\boldsymbol{g}_{A}$ and $H_{F}(B)$ is the algebra of tangential cohomologies. This homomorphism is trivial if there exists a flat $F$-connection in $A$. The transitive case was applied to TC-foliations in [K4] where we give an interpretation of the Chern-Weil homomorphism for the Lie algebroid $A(B, E)$ of a TC-foliation $(B, E)$; in particular, for foliations of left cosets of nonclosed Lie subgroups in a given Lie group. We have also given a family of examples of TC-foliations $(B, E)$ such that the homomorphism $h_{A(B, E)}$ is nontrivial and the algebroid $A(B, E)$ of this foliation is nonintegrable. As yet these examples have not been studied by the classical theory.

(2) If $L$ and $A$ are Lie algebroids over the same manifold $B, A$ is regular over $(B, F)$, $\operatorname{Im} \omega_{L} \subset F$ and $\lambda: F \rightarrow A$ is any connection in $A$, then $\nabla:=\lambda \circ \omega_{L}: L \rightarrow A$ is an $L$-connection in $A$. Indeed, $\omega_{A} \circ \nabla=\left(\omega_{A} \circ \lambda\right) \circ \omega_{L}=\omega_{L}$.

(3) Let $\pi: A \rightarrow L$ be a given epimorphism of Lie algebroids. We can always put it into an extension of Lie algebroids

$$
\text { e : } \quad 0 \rightarrow L^{\prime} \rightarrow A \stackrel{\pi}{\rightarrow} L \rightarrow 0
$$

where $L^{\prime}=\operatorname{ker} \pi$ has a structure of a Lie algebroid with the zero anchor. The right inverse of $\pi$, i.e. a linear homomorphism $\nabla: L \rightarrow A, \pi \circ \nabla=\mathrm{id}_{L}$ (called an e-connection or a connection in e) is an $L$-connection in $A$ since $\omega_{L}=\omega_{L} \circ \pi \circ \nabla=\omega_{A} \circ \nabla$. The sequence of global cross-sections induced by (2.3) gives the so-called extension of LieRinehart algebras which was examined by J. Huebschmann in [H1], [H2] (and earlier by N. Teleman [T1], [T2]). Note that they were also examined from different points of view by other authors and by the use of different terminology. For instance, these algebras were called Lie pseudoalgebras, Lie modules, etc., see [M3]. In [H2], [T2] the Chern-Weil homomorphism for extensions of Lie-Rinehart algebras is defined.

(4) Consider the short exact sequence of the jet bundles of a tangent bundle

$$
0 \rightarrow S^{k}(T B, T B) \rightarrow J^{k}(T B) \rightarrow J^{k-1}(T B) \rightarrow 0 .
$$

$J^{k}(T B)$ has a Lie algebroid structure [L] defined as follows: the anchor $\omega^{k}: J^{k}(T B) \rightarrow$ $T B$ is given by $\omega^{k}\left(j_{x}^{k} X\right)=X_{x}$, in Sec $J^{k}(T B)$ there exists a unique structure of a Lie algebra $\llbracket \cdot, \cdot \rrbracket$ with $\llbracket j^{k} X, j^{k} Y \rrbracket=j^{k}[X, Y]$. Hence we obtain the algebroid of the Lie grupoid of invertible holonomic $k$-jets of local diffeomorphisms of $B$; connections in this algebroid are called connections of rank $k$ on $B$. Splittings of the sequence (2.4) are called complete differentials of order $k$ on $B$. If we take $k=1$, we obtain the normal covariant 
derivative in the tangent bundle $T B$. Let us explain this phenomenon. The sequence (2.4) has the form $0 \rightarrow \operatorname{End}(T B) \stackrel{\iota}{\rightarrow} J^{1}(T B) \rightarrow T B \rightarrow 0, \iota(d f \otimes X)=j^{1}(f \cdot X)-f \cdot j^{1} X$, while a covariant derivative induced by the splitting $\nabla: T B \rightarrow J^{1}(T B)$ is defined by $\nabla_{X} Y=\left(j^{1} Y-\nabla Y\right)(X)$ (notice that $\left.j^{1} Y-\nabla Y \in \operatorname{End}(T B)\right)$, and conversely we have $\nabla Y=j^{1} Y-\nabla_{(\cdot)} Y$.

(5) [K. Mackenzie, [M4], [M5]] If

$$
N \longmapsto Q(B, H, q) \stackrel{\pi}{\rightarrow} P(B, G, p) \rightarrow 0
$$

is an extension of principal bundles (with respect to an extension of Lie groups $0 \rightarrow N \rightarrow$ $H \stackrel{\pi}{\rightarrow} G \rightarrow 0)$ and $\mathbf{e}: 0 \rightarrow K \rightarrow T Q_{/ H} \rightarrow T P_{/ G} \rightarrow 0$ is an extension which corresponds to Lie algebroids, then a connection in $\mathbf{e}$ is called a transversal connection in (2.5). There exists a one-to-one correspondence between these connections and $G$-equivariant connections in the Lie algebroid $T Q_{/ N}$ of the transversal principal $N$-bundle $Q(P, N, \pi)$.

(6) If Lie algebroids $L$ and $A$ are regular over the same foliated manifold $(B, F)$, then for their Whitney product $A \boxplus L$ we can observe the following fact: there exists a one-to-one correspondence between $L$-connections in $A$ and connections of the induced extension $\mathbf{e}_{L, A}: 0 \rightarrow \boldsymbol{g}_{A} \rightarrow A \boxplus L \stackrel{\pi}{\rightarrow} L \rightarrow 0$. In fact, $\nabla: L \rightarrow A \boxplus L, v \mapsto\left(\nabla^{1}(v), v\right)$, is a connection in this splitting if and only if $\omega_{A} \circ \nabla^{1}=\omega_{L}$, i.e. if $\nabla^{1}$ is an $L$-connection in $A$. Recall that the Whitney product (see [K1]) of regular algebroids $L$ and $A$ over the same foliated manifold $(B, F)$ is defined as a subbundle $A \boxplus L$ of the direct sum $A \oplus L$ such that fibres consist of pairs of vectors with equal anchors; a Lie bracket in the space of cross-sections is defined by coordinates and the anchor is given in an evident manner. This example shows that for a regular Lie algebroid the homomorphism $h_{L, A}$ constructed in our work can be expressed in terms of the Chern-Weil homomorphism of the extension $\mathbf{e}_{L, A}$ (generally given by N. Teleman and J. Huebschmann).

(7) Let $\nabla: L \rightarrow A$ be any $L$-connection in a regular Lie algebroid $A$ (with the Atiyah sequence $0 \rightarrow \boldsymbol{g} \rightarrow A \stackrel{\omega_{A}}{\rightarrow} F \rightarrow 0$ ) and let $\Omega_{\nabla}$ be its curvatute form defined below. It is easy to check that in the direct sum $\boldsymbol{g} \oplus L$ there exists a Lie algebroid structure such that $\omega_{L} \circ \mathrm{pr}_{2}: \boldsymbol{g} \oplus L \rightarrow \operatorname{Im}\left(\omega_{L}\right) \subset T B$ serves as the anchor and the Lie bracket in Sec $\boldsymbol{g} \oplus L$ is defined via the formula

$$
\llbracket\left(\nu_{1}, \xi_{1}\right),\left(\nu_{2}, \xi_{2}\right) \rrbracket=\left(\left[\nu_{1}, \nu_{2}\right]+\llbracket \nabla \xi_{1}, \nu_{2} \rrbracket+\llbracket \nu_{1}, \nabla \xi_{2} \rrbracket+\Omega_{\nabla}\left(\xi_{1}, \xi_{2}\right), \llbracket \xi_{1}, \xi_{2} \rrbracket\right) .
$$

The following is an extension of Lie algebroids

$$
\mathbf{e}_{\nabla}: 0 \rightarrow \boldsymbol{g} \rightarrow \boldsymbol{g} \oplus L \rightarrow L \rightarrow 0,
$$

$\boldsymbol{g} \oplus L$ and $L$ are of course over the same foliation $\operatorname{Im}\left(\omega_{L}\right)$.

(a) Clearly, $L$-connections in $A$ are in 1-1 correspondence to splittings of $\mathbf{e}_{\nabla}$ :

$$
\nabla+r \stackrel{\cong}{\mapsto} \lambda^{r}
$$

where $r: L \rightarrow \boldsymbol{g}$ and $\lambda^{r}: L \rightarrow \boldsymbol{g} \oplus L, \xi \mapsto(r \xi, \xi)$. It is important that flat connections correspond to flat splittings. (Remark: for a connection form of an $L$-connection $\nabla^{2}=$ $\nabla+r: L \rightarrow A$ we can take $\boldsymbol{g} \oplus L \rightarrow \boldsymbol{g},(\nu, \xi) \mapsto \nu-r(\xi)$, the connection form corresponding to the suitable splitting $\lambda^{r}$ of $\mathbf{e}_{\nabla}$.)

(b) If $\nabla^{2}=\nabla+r$ is another $L$-connection in $A$ and $\mathbf{e}_{\nabla^{2}}$ is the extension defined via $\nabla^{2}$ then the extensions are equivalent: $\rho: \boldsymbol{g} \oplus L \rightarrow(\boldsymbol{g} \oplus L)^{(2)},(\nu, \xi) \mapsto(\nu-r \xi, \xi)$, is an isomorphism of extensions of Lie algebroids. 
The above results generalize the standard case of $L=\operatorname{Im}\left(\omega_{A}\right)=F$ for regular Lie algebroids [K8] (and the earlier one corresponding to transitive Lie algebroids [M1], [K3]).

2.2. The curvature of an $L$-connection and exterior covariant derivative. With a Lie algebroid $L$ and a vector bundle $\mathfrak{f}$ on the same manifold $B$ we can connect the space of alternating forms $\Omega_{L}(B ; \mathfrak{f})=\oplus^{n} \Omega_{L}^{n}(B ; \mathfrak{f})$, where $\Omega_{L}^{n}(B ; \mathfrak{f})=\operatorname{Sec} \bigwedge^{n} L^{*} \otimes \mathfrak{f}$. For $\Theta_{i} \in$ $\Omega_{L}^{q_{i}}\left(B ; \mathfrak{f}^{i}\right)$ and a multilinear homomorphism of vector bundles $\varphi: \mathfrak{f}^{1} \times \ldots \times \mathfrak{f}^{k} \rightarrow \mathfrak{f}$ we assume that $\varphi_{*}\left(\Theta_{1}, \ldots, \Theta_{k}\right) \in \Omega_{L}^{q_{1}+\ldots+q_{k}}(B ; \mathfrak{f})$ is given by the well-known formula

$$
\begin{aligned}
& \varphi_{*}\left(\Theta_{1}, \ldots, \Theta_{k}\right)\left(x ; v_{1}, \ldots, v_{m}\right) \\
& =\frac{1}{q_{1} ! \cdot \ldots \cdot q_{k} !} \sum_{\sigma} \operatorname{sgn} \sigma \cdot \varphi\left(x ; \Theta_{1}\left(x ; v_{\sigma(1)} \wedge \ldots\right), \ldots, \Theta_{k}\left(x ; \ldots \wedge v_{\sigma(m)}\right)\right) .
\end{aligned}
$$

In the case of standard homomorphisms $\varphi$ of the form $\bigvee^{k}: \mathfrak{f} \times \ldots \times \mathfrak{f} \rightarrow \bigvee^{k} \mathfrak{f}$ (symmetric power), $\langle\cdot, \cdot\rangle: \bigvee^{k} \mathfrak{f}^{*} \times \bigvee^{k} \mathfrak{f} \rightarrow \mathbb{R}$ (duality) etc., it is better to use the notation from [G-H-V], the form $\varphi_{*}\left(\Theta_{1}, \ldots, \Theta_{k}\right)$ will be denoted by $\Theta_{1} \vee \ldots \vee \Theta_{k},\left\langle\Theta_{1}, \Theta_{2}\right\rangle$, etc. For real forms $\Omega_{L}(B)$ there exists a derivative $d_{L}$ defined by the known formula [MR], [K2], [M1]

$$
\begin{aligned}
\left(d_{L} \theta\right)\left(\xi_{0}, \ldots, \xi_{k}\right)= & \sum_{j=0}^{k}(-1)^{j}\left(\omega_{L} \circ \xi_{i}\right)\left(\theta\left(\xi_{0}, \ldots \hat{\jmath} \ldots, \xi_{k}\right)\right) \\
& +\sum_{i<j}(-1)^{i+j} \theta\left(\llbracket \xi_{i}, \xi_{j} \rrbracket, \xi_{0}, \ldots \hat{\imath} \ldots \hat{\jmath} \ldots, \xi_{k}\right) .
\end{aligned}
$$

Definition 2.5. Assume that $L$ and $A$ are Lie algebroids on $B$, and $A$ is regular with Atiyah sequence $0 \rightarrow \boldsymbol{g}_{A} \rightarrow A \rightarrow F \rightarrow 0$. By the curvature form of an $L$-connection $\nabla: L$ $\rightarrow A$ we shall mean the 2 -form $\Omega_{\nabla} \in \Omega_{L}^{2}\left(B ; \boldsymbol{g}_{A}\right)$ defined by

$$
\Omega_{\nabla}(\xi, \eta)=\llbracket \nabla \circ \xi, \nabla \circ \eta \rrbracket-\nabla \circ \llbracket \xi, \eta \rrbracket, \quad \xi, \eta \in \operatorname{Sec} L,
$$

$\left(\Omega_{\nabla}(\xi, \eta)\right.$ is a cross-section of the bundle $\boldsymbol{g}_{A}$ since $\left.\omega_{A} \circ \Omega_{\nabla}(\xi, \eta)=0\right)$.

With a given $L$-connection $\nabla: L \rightarrow A$ we can connect the so-called exterior covariant derivative $\tilde{\nabla}: \Omega_{L}^{*}\left(B ; \boldsymbol{g}_{A}\right) \rightarrow \Omega_{L}^{*+1}\left(B ; \boldsymbol{g}_{A}\right)$ defined for a $k$-form $\Theta$ by the formula $\left(\xi_{i} \in\right.$ $\operatorname{Sec} L)$

$$
\begin{aligned}
(\tilde{\nabla} \Theta)\left(\xi_{0}, \ldots, \xi_{k}\right)= & \sum_{j=0}^{k}(-1)^{j} \llbracket \nabla \circ \xi_{j}, \Theta\left(\xi_{0}, \ldots \hat{\jmath} \ldots, \xi_{k}\right) \rrbracket \\
& +\sum_{i<j}(-1)^{i+j} \Theta\left(\llbracket \xi_{i}, \xi_{j} \rrbracket, \xi_{0}, \ldots \hat{\imath} \ldots \hat{\jmath} \ldots, \xi_{k}\right) .
\end{aligned}
$$

It is standard to check the so-called Bianchi identity

$$
\tilde{\nabla}\left(\Omega_{\nabla}\right)=0 .
$$

Immediately from definition $(2.7)$ we obtain $\tilde{\nabla} \nu \in \Omega_{L}^{1}\left(B ; \boldsymbol{g}_{A}\right),(\tilde{\nabla} \nu)(\xi)=\llbracket \nabla \circ \xi, \nu \rrbracket$, for $\nu \in \operatorname{Sec} \boldsymbol{g}_{A}$ and $\xi \in \operatorname{Sec} L$. If $\theta \in \Omega_{L}(B)$ and $\nu \in \operatorname{Sec} \boldsymbol{g}_{A}$, then

$$
\tilde{\nabla}(\nu \cdot \theta)=\tilde{\nabla} \nu \wedge \theta+\nu \cdot d_{L} \theta .
$$


Indeed, according to (2.2) and (2.1), for $\theta \in \Omega_{L}^{k}(B)$ and $\xi_{i} \in \operatorname{Sec} L$, we have

$$
\begin{aligned}
& \left(\tilde{\nabla} \nu \wedge \theta+\nu \cdot d_{L} \theta\right)\left(\xi_{0}, \ldots, \xi_{k}\right) \\
& =\sum_{j=0}^{k}(-1)^{j} \llbracket \nabla \circ \xi_{j}, \nu \rrbracket \cdot \theta\left(\xi_{0}, \ldots \hat{\jmath} \ldots, \xi_{k}\right) \\
& +\sum_{j=0}^{k}(-1)^{j}\left(\omega_{L} \circ \xi_{j}\right)\left(\theta\left(\xi_{0}, \ldots \hat{\jmath} \ldots, \xi_{k}\right)\right) \cdot \nu+\sum_{i<j}(-1)^{j} \theta\left(\llbracket \xi_{i}, \xi_{j} \rrbracket, \xi_{0}, \ldots \hat{\imath} \ldots \hat{\jmath} \ldots, \xi_{k}\right) \cdot \nu \\
& =\sum_{j=0}^{k}(-1)^{j} \llbracket \nabla \circ \xi_{j}, \theta\left(\xi_{0}, \ldots \hat{\jmath} \ldots, \xi_{k}\right) \cdot \nu \rrbracket+\sum_{i<j}(-1)^{i+j} \theta\left(\llbracket \xi_{i}, \xi_{j} \rrbracket, \xi_{0}, . . \hat{\imath} \ldots \hat{\jmath} \ldots, \xi_{k}\right) \cdot \nu \\
& =\nabla(\nu \cdot \theta)\left(\xi_{0}, \ldots, \xi_{k}\right) .
\end{aligned}
$$

A trivial verification again shows that

$$
\begin{aligned}
& \varphi_{*}\left(\nu_{1} \cdot \theta_{1}, \ldots, \nu_{k} \cdot \theta_{k}\right)=\varphi\left(\nu_{1}, \ldots, \nu_{k}\right) \cdot \theta_{1} \wedge \ldots \wedge \theta_{k} \\
& \varphi_{*}\left(\nu_{1}, \ldots, \tilde{\nabla} \nu_{i}, \ldots, \nu_{k}\right) \wedge \theta_{1} \wedge \ldots \wedge \theta_{k} \\
& =(-1)^{q_{1}+\ldots+q_{i-1}} \varphi_{*}\left(\nu_{1} \cdot \theta_{1}, \ldots, \nabla \nu_{i} \wedge \theta_{i}, \ldots, \nu_{k} \cdot \theta_{k}\right)
\end{aligned}
$$

for $\nu_{i} \in \operatorname{Sec} \boldsymbol{g}_{A}, \theta_{i} \in \Omega_{L}^{q_{i}}(B), \varphi: \boldsymbol{g}_{A} \times \ldots \times \boldsymbol{g}_{A} \rightarrow \mathbb{R}$.

2.3. Representations and their invariant cross-sections. Let $\mathfrak{f}$ be a vector bundle on a manifold $B$. By a representation (action) of a Lie algebroid $A$ on $\mathfrak{f}$ we mean a strong homomorphism of Lie algebroids $R: A \rightarrow A(\mathfrak{f})$ [M1]. A cross-section $\nu \in \operatorname{Sec} \mathfrak{f}$ is called $R$-invariant (or briefly invariant - unless it leads to confusion) if $(R \circ \xi)(\nu)=0$ for all $\xi \in A$. If two invariant cross-sections are equal at a point, then they are equal along the leaf of the characteristic foliation which contains this point. An important example of a representation for a regular Lie algebroid $A$ is the so-called adjoint representation $\operatorname{ad}_{A}: A \rightarrow A\left(\boldsymbol{g}_{A}\right)$ defined so that $\left(\operatorname{ad}_{A} \circ \xi\right)(\eta)=\llbracket \xi, \eta \rrbracket, \xi \in \operatorname{Sec} A, \eta \in \operatorname{Sec} \boldsymbol{g}_{A}$.

A single representation $R$ determines (as in the case of a representation of a Lie algebra on a vector space) new representations on associated bundles [K4], for example the contragredient representation $R^{\natural}$ on the dual bundle $\mathfrak{f}^{*}$, the symmetric power $\bigvee^{k} R$ on the symmetric product $\bigvee^{k} \mathfrak{f}, \operatorname{Hom}^{k}(R)$ on the bundle of $k$-linear homomorphisms $\operatorname{Hom}^{k}(\mathfrak{f} ; \mathbb{R})$. The last one is given by

$$
\begin{aligned}
\left(\operatorname{Hom}^{k}(R) \circ \xi\right)(\varphi)\left(v_{1}, \ldots, v_{k}\right) & \\
& =\left(\omega_{L} \circ \xi\right)\left(\varphi\left(v_{1}, \ldots, v_{k}\right)\right)-\sum_{i=1}^{k} \varphi\left(v_{1}, \ldots,(R \circ \xi)\left(\nu_{i}\right), \ldots, v_{k}\right),
\end{aligned}
$$

$\varphi: \mathfrak{f} \times \ldots \times \mathfrak{f} \rightarrow \mathbb{R}, \nu_{i} \in \operatorname{Sec} \mathfrak{f}$.

LEMMA 2.6. Let $\varphi: \boldsymbol{g}_{A} \times \ldots \times \boldsymbol{g}_{A} \rightarrow \mathbb{R}$ be a $\mathrm{Hom}^{k}\left(\operatorname{ad}_{A}\right)$-invariant cross-section. Then for $\Theta_{i} \in \Omega_{A}^{q_{i}}\left(B ; \boldsymbol{g}_{A}\right)$ the following equality holds:

$$
d_{A}\left(\varphi_{*}\left(\Theta_{1}, \ldots, \Theta_{k}\right)\right)=\sum_{i=1}^{k}(-1)^{q_{1}+\ldots+q_{i-1}} \varphi_{*}\left(\Theta_{1}, \ldots, \tilde{\nabla} \Theta_{i}, \ldots, \Theta_{k}\right) .
$$


Proof. Observe that for forms of degree zero, i.e. cross-sections $\nu_{i} \in \operatorname{Sec} \boldsymbol{g}_{A}$, the formula (2.13) can be written as follows:

$$
d_{A}\left(\varphi\left(\nu_{1}, \ldots, \nu_{k}\right)\right)=\sum_{i=1}^{k} \varphi_{*}\left(\nu_{1}, \ldots, \tilde{\nabla} \nu_{i}, \ldots, \nu_{k}\right), \quad \nu_{i} \in \operatorname{Sec} \boldsymbol{g}_{A}
$$

In view of (2.12) (for $R=\operatorname{ad}_{A}$ ), from definition (2.6) we have

$$
\begin{aligned}
d_{A}\left(\varphi\left(\nu_{1}, \ldots, \nu_{k}\right)\right)(\xi) & =\left(\omega_{A} \circ \xi\right)\left(\varphi\left(\nu_{1}, \ldots, \nu_{k}\right)\right)=\sum_{i=1}^{k} \varphi\left(\nu_{1}, \ldots, \llbracket \nabla \circ \xi, \nu_{i} \rrbracket, \ldots, \nu_{k}\right) \\
& =\sum_{i=1}^{k} \varphi\left(\nu_{1}, \ldots, \tilde{\nabla} \nu_{i}(\xi), \ldots, \nu_{k}\right)=\sum_{i=1}^{k} \varphi_{*}\left(\nu_{1}, \ldots, \tilde{\nabla} \nu_{1}, \ldots, \nu_{k}\right)(\xi)
\end{aligned}
$$

for $\xi \in \operatorname{Sec} A$.

It is sufficient to prove the equality (2.13) for forms $\Theta_{i}=\nu_{i} \cdot \theta_{i}$ where $\nu_{i} \in \operatorname{Sec} \boldsymbol{g}_{A}$, $\theta_{i} \in \Omega_{A}^{q_{i}}(B)$. From the equalities (2.9)-(2.11) and (2.14) we obtain

$$
\begin{aligned}
d_{A}\left(\varphi_{*}\left(\nu_{1} \cdot \theta_{1}, \ldots, \nu_{k} \cdot \theta_{k}\right)\right) & \\
= & d_{A}\left(\varphi\left(\nu_{1}, \ldots, \nu_{k}\right)\right) \wedge \theta_{1} \wedge \ldots \wedge \theta_{k}+\varphi\left(\nu_{1}, \ldots, \nu_{k}\right) \cdot d_{A}\left(\theta_{1} \wedge \ldots \wedge \theta_{k}\right) \\
= & \sum_{i=1}^{k} \varphi_{*}\left(\nu_{1}, \ldots, \tilde{\nabla} \nu_{k}, \ldots, \nu_{k}\right) \wedge \theta_{1} \wedge \ldots \wedge \theta_{k} \\
& +\varphi\left(\nu_{1}, \ldots, \nu_{k}\right) \sum_{i=1}^{k}(-1)^{q_{1}+\ldots+q_{i-1}} \theta_{1} \wedge \ldots \wedge d_{A} \theta_{i} \wedge \ldots \wedge \theta_{k} \\
= & \sum_{i=1}^{k}(-1)^{q_{1}+\ldots+q_{-1}} \varphi_{*}\left(\nu_{1} \cdot \theta_{1}, \ldots, \tilde{\nabla} \nu_{1} \wedge \theta_{i}+\nu_{1} \cdot d_{A} \theta_{i}, \ldots, \nu_{k} \cdot \theta_{k}\right) \\
= & \sum_{i=1}^{k}(-1)^{q_{1}+\ldots+q_{i-1}} \varphi_{*}\left(\nu_{1} \cdot \theta_{1}, \ldots, \tilde{\nabla}\left(\nu_{i} \theta_{i}\right), \ldots, \nu_{k} \cdot \theta_{k}\right) .
\end{aligned}
$$

By definition, the contragredient representation $R^{\natural}$ of $R: A \rightarrow A(\mathfrak{f})$ is such that $R^{\natural} \circ \xi$ is a covariant differential operator in the dual bundle $\mathfrak{f}^{*}$, satisfying $\left(R^{\natural} \circ \xi\right)(\varphi)(\nu)=$ $\left(\omega_{A} \circ \xi\right)\langle\varphi, \nu\rangle-\langle\varphi,(R \circ \xi) \nu\rangle$. The symmetric product $\bigvee^{k} R$ on $\bigvee^{k} \mathfrak{f}$ is defined as the one for which $\left(\left(\bigvee^{k} R\right) \circ \xi\right)\left(\nu^{1} \vee \ldots \vee \nu^{k}\right)=\sum_{i=1}^{k} \nu^{1} \vee \ldots \vee(R \circ \xi)\left(\nu^{i}\right) \vee \ldots \vee \nu^{k}, \nu^{i} \in$ Sec f. If $\gamma^{1} \in \operatorname{Sec} \bigvee^{k} \mathfrak{f}$ and $\gamma^{2} \in \operatorname{Sec} \bigvee^{l} \mathfrak{f}$ are invariant cross-sections with respect to representations $\bigvee^{k} R$ and $\bigvee^{l} R$, respectively, then their symmetric product $\gamma^{1} \vee \gamma^{2} \in \operatorname{Sec} \bigvee^{k+l} \mathfrak{f}$ is also invariant [K4]. This implies that the space of invariant cross-sections of all symmetric powers of $\mathfrak{f}$ forms an algebra. In the cited work it is also proved (using the permanent of a matrix) that the symmetric product of the contragredient representation of $R, \bigvee^{k} R^{\natural}$, is given by the formula

$$
\begin{aligned}
& \left\langle\left(\left(\vee^{k} R^{\natural}\right) \circ \xi\right)(\Gamma), \nu^{1} \vee \ldots \vee \nu^{k}\right\rangle \\
& =\left(\omega_{A} \circ \xi\right)\left\langle\Gamma, \nu^{1} \vee \ldots \vee \nu^{k}\right\rangle-\sum_{i=1}^{k}\left\langle\Gamma, \nu^{1} \vee \ldots \vee \nu^{k}(R \circ \xi)\left(\nu^{i}\right) \vee \ldots \vee \nu^{k}\right\rangle,
\end{aligned}
$$

$\Gamma \in \operatorname{Sec} \bigvee^{k} \mathfrak{f}^{*}, \nu^{i} \in \operatorname{Sec} \mathfrak{f}$ 


\section{The Chern-Weil homomorphism}

3.1. Classical theory for principal bundles. The Chern-Weil homomorphism for a $G$ principal bundle $P(B, G, \pi)$ is a homomorphism of algebras

$$
h_{P}:\left(\bigvee \mathfrak{g}^{*}\right)_{I} \rightarrow H_{d R}(B) .
$$

The domain

$$
I(G):=\left(\bigvee \mathfrak{g}^{*}\right)_{I}
$$

is the space of polynomials $\Gamma: \mathfrak{g} \times \ldots \times \mathfrak{g} \rightarrow \mathbb{R}$ invariant with respect to the adjoint representation $A d_{G}$ of $G$ on $\bigvee \mathfrak{g}^{*}$. The homomorphism $h_{P}$ is defined by the formula

$$
h_{P}(\Gamma)=\left[\chi_{P}(\Gamma)\right]
$$

where

$$
\pi^{*}\left(\chi_{P}(\Gamma)\right)=\frac{1}{k !}[\langle\Gamma, \Omega \vee \ldots \vee \Omega\rangle] \quad\left(\text { for } \Gamma \in\left(\bigvee^{k} \mathfrak{g}^{*}\right)_{I}\right)
$$

and $\Omega \in \Omega^{2}(P, \mathfrak{g})$ is the curvature form of some connection $H \subset T P$ and $\Omega \vee \ldots \vee \Omega$ denotes the standard skew-product of forms with symmetric multiplication of the values [G-H-V].

Equivalently, we can define $h_{P}$ directly on $B$ without using the lifting to $P$; we can use the Atiyah sequence

$$
0 \rightarrow P \times_{G} \mathfrak{g} \rightarrow T P_{/ G} \stackrel{\omega}{\rightarrow} T B \rightarrow 0
$$

of $P$ instead the lifting. Let $\nabla: T B \rightarrow T P_{/ G}$ be the splitting of this sequence corresponding to a connection $H$,

$$
0 \rightarrow P \times_{G} \mathfrak{g} \rightarrow T P / G \underset{\nabla}{\stackrel{\omega}{\rightleftarrows}} T B \rightarrow 0 .
$$

Recall that $P \times_{G} \mathfrak{g}$ is a Lie algebra bundle (LAB); for $z \in P_{\mid x}$ we have an isomorphism of Lie algebras

$$
\hat{z}: \mathfrak{g} \rightarrow\left(P \times_{G} \mathfrak{g}\right)_{\mid x}, \quad v \mapsto\left(A_{z}\right)_{* e}(v),
$$

$A_{z}: G \rightarrow P, a \mapsto z a$, provided that $\mathfrak{g}$ is the right Lie algebra of $G$ (not left!). Next, we make the following observations:

- each invariant polynomial $\Gamma \in\left(\bigvee^{k} \mathfrak{g}^{*}\right)_{I}$ determines a symmetric $k$-linear homomorphism

$$
\tilde{\Gamma}: P \times_{G} \mathfrak{g} \times \ldots \times P \times_{G} \mathfrak{g} \rightarrow B \times \mathbb{R}
$$

(i.e. a cross-section of $\left.\bigvee^{k}\left(P \times_{G} \mathfrak{g}\right)^{*}\right)$ defined by

$$
\tilde{\Gamma}_{x}=\bigvee^{k}\left(\hat{z}^{-1}\right)^{*}(\Gamma), \quad z \in P_{\mid x},
$$

(the formula is independent of the choice of $z$ since $\Gamma$ is invariant).

- the curvature form $\Omega=\pi^{*}\left(\Omega_{b}\right)$, where $\Omega_{b} \in \Omega^{2}\left(B ; P \times_{G} \mathfrak{g}\right)$ is defined by

$$
\Omega_{b}(X, Y)=\llbracket \nabla X, \nabla Y \rrbracket-\nabla[X, Y] .
$$


We have the equality

$$
\langle\Gamma, \Omega \vee \ldots \vee \Omega\rangle=\pi^{*}\left\langle\tilde{\Gamma}, \Omega_{b} \vee \ldots \vee \Omega_{b}\right\rangle
$$

and

$$
\chi_{P}(\Gamma)=\frac{1}{k !}\left\langle\tilde{\Gamma}, \Omega_{b} \vee \ldots \vee \Omega_{b}\right\rangle
$$

therefore

$$
h_{P}(\Gamma)=\frac{1}{k !}\left[\left\langle\tilde{\Gamma}, \Omega_{b} \vee \ldots \vee \Omega_{b}\right\rangle\right] .
$$

Now we describe the space

$$
\left\{\tilde{\Gamma} ; \Gamma \in\left(\bigvee^{k} \mathfrak{g}^{*}\right)_{I}\right\}
$$

in terms of the Lie algebroid $T P_{/ G}$. We notice that $\left(\bigvee^{k} \mathfrak{g}^{*}\right)_{I}$ depends on $G$, not only on its Lie algebra $\mathfrak{g}=\mathfrak{g} l(G)$ (unless $G$ is a connected Lie group) but in $T P_{/ G}$ we have no information about the structural Lie group $G$. We need to use the adjoint representation of the Lie algebroid $T P_{/ G}$ in the vector bundle $P \times_{G} \mathfrak{g}$

$$
\operatorname{ad}_{T P_{/ G}}: T P_{/ G} \rightarrow A\left(P \times_{G} \mathfrak{g}\right)
$$

defined by

$$
\operatorname{ad}_{T P_{/ G}}(\xi)(\nu)=\llbracket \xi, \nu \rrbracket, \xi \in \operatorname{Sec}\left(T P_{/ G}\right), \nu \in \operatorname{Sec}\left(P \times_{G} \mathfrak{g}\right) .
$$

The representation $\operatorname{ad}_{T P_{/ G}}$ induces representations of $T P_{/ G}$ on associated vector bundles, among others, on $\bigvee^{k}\left(P \times_{G} \mathfrak{g}\right)^{*}$, by

$$
\operatorname{ad}_{T P_{/ G}}(\xi)(\varphi)\left(\nu_{1}, \ldots, \nu_{k}\right)=(\omega \xi)\left(\varphi\left(\nu_{1}, \ldots, \nu_{k}\right)\right)-\sum_{i} \varphi\left(\nu_{1}, \ldots, \llbracket \xi, \nu_{i} \rrbracket, \ldots, \nu_{k}\right),
$$

where $\varphi \in \operatorname{Sec} \bigvee^{k}\left(P \times_{G} \mathfrak{g}\right)^{*}$, equivalently $\varphi$ is a symmetric tensor $\varphi:\left(P \times_{G} \mathfrak{g}\right) \times \ldots \times$ $\left(P \times_{G} \mathfrak{g}\right) \rightarrow B \times \mathbb{R} . \varphi$ is called invariant if $\operatorname{ad}_{T P_{/ G}}(\xi)(\varphi)=0$ for all $\xi \in \operatorname{Sec}\left(T P_{/ G}\right)$. We define the algebra of invariant tensors

$$
I\left(T P_{/ G}\right)=\bigoplus^{k}\left(\operatorname{Sec} \bigvee^{k}\left(P \times_{G} \mathfrak{g}\right)^{*}\right)_{I}
$$

TheOrem 3.1 (Kubarski [K4]; Belko [B1], [B2]). The homomorphism

$$
\rho:\left(\bigvee \mathfrak{g}^{*}\right)_{I} \rightarrow I\left(T P_{/ G}\right), \Gamma \mapsto \tilde{\Gamma},
$$

is a monomorphism of algebras and is an isomorphism if $P$ is connected ( $G$ may be disconnected!).

By the Chern-Weil homomorphism of the Lie algebroid $T P_{/ G}$ we mean the homomorphism

$$
h_{T P_{/ G}}: I\left(T P_{/ G}\right) \rightarrow H_{d R}(M), \quad \varphi \mapsto \frac{1}{k !}\left[\left\langle\varphi, \Omega_{b} \vee \ldots \vee \Omega_{b}\right\rangle\right]
$$

(for $\varphi$ of degree $k$ ) where $\Omega_{b} \in \Omega^{2}\left(B ; P \times_{G} \mathfrak{g}\right)$ is the curvature of any connection $\nabla: T B$ $\rightarrow T P_{/ G}$ in the Lie algebroid $T P_{/ G}$.

We have the commuting diagram 


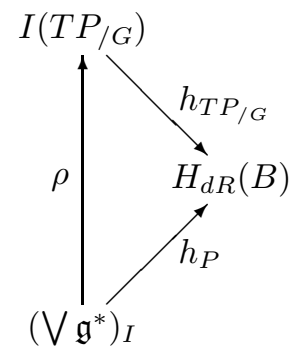

Therefore, if $P$ is connected, its Chern-Weil homomorphism is equivalent to the ChernWeil homomorphism of the Lie algebroid $T P_{/ G}$.

3.2. The Chern-Weil homomorphism for a pair of Lie algebroids. Assume that $L$ and $A$ are Lie algebroids on a manifold $B$, and $A$ is regular (assumptions as in Definition 2.5). We shall construct a characteristic homomorphism which will somehow measure obstructions to existence of a flat $L$-connection in $A$. The Chern-Weil homomorphism in the case of an integrable Lie algebroid $A=T P_{/ G}$ is closely connected with the one obtained recently by R. L. Fernandes [F2].

3.2.1. Construction. Let $x \in B$ be a fixed point. Since $\bigwedge^{\mathrm{ev}} L_{\mid x}^{*}:=\bigoplus_{k \geq 0} \bigwedge^{2 k} L_{\mid x}^{*}$ is a symmetric algebra, there exists precisely one homomorphism of algebras (see [G, p. 192])

such that

$$
\chi_{\nabla, x}: \bigvee\left(g_{A}\right)_{\mid x}^{*} \rightarrow \bigoplus_{k \geq 0} \bigwedge^{2 k} L_{\mid x}^{*}
$$

1. $\chi_{\nabla, x}(1)=1$,

2. $\chi_{\nabla, x}(\Gamma)=\left\langle\Gamma, \Omega_{\nabla x}\right\rangle, \quad \Gamma \in\left(\boldsymbol{g}_{A}\right)_{\mid x}^{*}$.

Lemma 3.2. For $\Gamma \in \bigvee^{k}\left(\boldsymbol{g}_{A}\right)_{\mid x}^{*}$ we have the equality

$$
\chi_{\nabla, x}(\Gamma)=\frac{1}{k !} \cdot\langle\Gamma, \underbrace{\Omega_{\nabla x} \vee \ldots \vee \Omega_{\nabla x}}_{k \text { times }}\rangle .
$$

Proof. We give an elementary proof using properties of permanents of matrices. Another proof, using a tensor algebra $\bigotimes\left(\boldsymbol{g}_{A}\right)_{\mid x}^{*}$ and its relation to a symmetric algebra $[\mathrm{G}$, pp.91, 193], is similar to the proof of Lemma 4.1.1 in [K4]. It is sufficient to show the equality (3.2) for $\Gamma=\Gamma_{1} \vee \ldots \vee \Gamma_{k}, \Gamma_{i} \in\left(\boldsymbol{g}_{A}\right)_{\mid x}^{*}$ :

$$
\begin{aligned}
\frac{1}{k !} \cdot\left\langle\Gamma_{1} \vee\right. & \left.\ldots \vee \Gamma_{k}, \Omega_{\nabla x} \vee \ldots \vee \Omega_{\nabla x}\right\rangle\left(v_{1} \wedge \ldots \wedge v_{2 k}\right) \\
& =\frac{1}{k !} \cdot\left\langle\Gamma_{1} \vee \ldots \vee \Gamma_{k}, \frac{1}{2^{k}} \sum_{\sigma} \operatorname{sgn} \sigma \cdot \Omega_{\nabla x}\left(v_{\sigma_{1}} \wedge v_{\sigma_{2}}\right) \vee \ldots \vee \Omega_{\nabla x}\left(v_{\sigma_{2 k-1}} \wedge v_{\sigma_{2 k}}\right)\right\rangle \\
& =\frac{1}{k !} \cdot \frac{1}{2^{k}} \sum_{\sigma} \operatorname{sgn} \sigma \cdot \operatorname{perm}\left[\left\langle\Gamma_{i}, \Omega_{\nabla x}\left(v_{\sigma_{2 j-1}} \wedge v_{\sigma_{2 j}}\right)\right\rangle\right]_{i, j=1, \ldots, k} \\
& =\frac{1}{k !} \cdot \frac{1}{2^{k}} \sum_{\sigma} \operatorname{sgn} \sigma \cdot k ! \cdot\left\langle\Gamma_{1}, \Omega_{\nabla x}\left(v_{\sigma_{1}} \wedge v_{\sigma_{2}}\right)\right\rangle \cdot \ldots \cdot\left\langle\Gamma_{k}, \Omega_{\nabla x}\left(v_{\sigma_{2 k-1}} \wedge v_{\sigma_{2 k}}\right)\right\rangle \\
& =\left\langle\Gamma_{1}, \Omega_{\nabla x}\right\rangle \wedge \ldots \wedge\left\langle\Gamma_{k}, \Omega_{\nabla x}\right\rangle\left(v_{1} \wedge \ldots \wedge v_{2 k}\right) \\
& =\chi \nabla, x \\
& \left.\chi \Gamma_{1} \vee \ldots \vee \Gamma_{k}\right)\left(v_{1} \wedge \ldots \wedge v_{2 k}\right) .
\end{aligned}
$$


For a fixed integer number $k \geq 0$, the family of homomorphisms $\chi_{\nabla, x}$ determines a strong homomorphism of vector bundles

$$
\chi_{\nabla}^{k}: \bigvee^{k} \boldsymbol{g}_{A}^{*} \rightarrow \bigwedge^{2 k} L^{*}
$$

such that, for $\Gamma \in \operatorname{Sec} \bigvee^{k} \boldsymbol{g}_{A}^{*}$, we obtain the equality $\chi_{\nabla}^{k} \circ \Gamma=\frac{1}{k !} \cdot\left\langle\Gamma, \Omega_{\nabla} \vee \ldots \vee \Omega_{\nabla}\right\rangle$, which, in particular, implies smoothness of the constructed homomorphism. Finally, we have the homomorphism of $C^{\infty}(B)$-modules

$$
\chi_{\nabla}: \bigoplus_{k \geq 0} \operatorname{Sec} \bigvee^{k} \boldsymbol{g}_{A}^{*} \rightarrow \Omega_{L}^{\mathrm{ev}}(B),
$$

which restricted to the algebra of invariant cross-sections $I(A)$ gives the homomorphism $\chi_{\nabla, I}$. The forms from its image are closed. In fact, a cross-section $\Gamma$ determines, in a standard way, a multilinear map $\tilde{\Gamma}: g_{A} \times \ldots \times g_{A} \rightarrow \mathbb{R}$. Then $\left\langle\Gamma, \Theta_{1} \vee \ldots \vee \Theta_{k}\right\rangle=$ $\tilde{\Gamma}_{*}\left(\Theta_{1}, \ldots, \Theta_{k}\right)$. If $\Gamma$ is invariant, then so is $\tilde{\Gamma}$. Hence, in view of the Bianchi identity (2.8) and formula (2.13), the form $\chi_{\nabla}^{k} \circ \Gamma$ is closed. This gives a homomorphism of algebras which acts into cohomologies $H_{L}(B)$

$$
h_{\nabla}: I(A) \rightarrow \operatorname{ker} d_{L} \rightarrow H_{L}(B) .
$$

All we need is to show that this homomorphism is independent of the choice of an $L$ connection $\nabla$. This will imply that $h_{\nabla}$ is a characteristic object for a pair of Lie algebroids $(L, A)$ on the same manifold.

3.2.2. Independence of the choice of an $L$-connection $\nabla$. One of the ways to show that the characteristic homomorphism $h_{\nabla}$ does not depend on the choice of the connection needs the use of nonstrong homomorphisms between two Lie algebroids, the notion of homotopy joining such homomorphisms, and the fact that homotopic homomorphisms induce the same mappings on cohomology. Let $\left(A, \llbracket \cdot, \cdot \rrbracket, \omega_{A}\right)$ and $\left(A^{\prime}, \llbracket \cdot, \cdot \rrbracket^{\prime}, \omega_{A^{\prime}}\right)$ be two Lie algebroids (not necessarily regular) on manifolds $B$ and $B^{\prime}$, respectively. By a homomorphism between them [M1], [K4], [K6], we mean a homomorphism of vector bundles $H: A \rightarrow A^{\prime}$, say, over $f: B \rightarrow B^{\prime}$, such that (1) $\omega_{A^{\prime}} \circ H=f_{*} \circ \omega_{A}$, (2) for arbitrary crosssections $\xi, \eta \in \operatorname{Sec} A$ with $H$-decompositions $H \circ \xi=\sum_{i} f^{i} \cdot\left(\sigma_{i} \circ f\right), H \circ \eta=\sum_{j} g^{j} \cdot\left(\tau_{j} \circ f\right)$, $f^{i}, g^{j} \in C^{\infty}(B), \sigma_{i}, \tau_{j} \in \operatorname{Sec} A^{\prime}$, we have

$$
\begin{aligned}
& H \circ \llbracket \xi, \eta \rrbracket \\
& =\sum_{i, j} f^{i} \cdot g^{j} \cdot \llbracket \sigma_{i}, \tau_{j} \rrbracket^{\prime} \circ f+\sum_{j}\left(\omega_{A} \circ \xi\right)\left(g^{j}\right) \cdot \tau_{j} \circ f-\sum_{i}\left(\omega_{A} \circ \eta\right)\left(f^{i}\right) \cdot \sigma_{i} \circ f .
\end{aligned}
$$

Although this notion seems to be complicated it is quite obvious from the Lie groupoid point of view (a nonstrong homomorphism of groupoids preserves source and target and partial multiplication; after infinitesimal linearization we obtain a homomorphism of algebroids). Notice that in the case of Lie algebroids on the same manifold $B$, a strong homomorphism is just a homomorphism of bundles which commutes with anchors and Lie brackets. Let $H$ and $H^{\prime}$ be homomorphisms $A \rightarrow A^{\prime}$. By a homotopy [K6] joining $H$ to $H^{\prime}$ we mean a homomorphism of Lie algebroids $\bar{H}: T \mathbb{R} \times A \rightarrow A^{\prime}$ such that $\bar{H}\left(\theta_{0}, \cdot\right)=H$ and $\bar{H}\left(\theta_{1}, \cdot\right)=H^{\prime}$ where $\theta_{0}$ and $\theta_{1}$ are null vectors tangent to $\mathbb{R}$ at 0 and 1 , respectively. The homotopy $\bar{H}$ induces a chain homotopy operator $k: \Omega_{A^{\prime}}^{*}\left(B^{\prime}\right) \rightarrow \Omega_{A}^{*-1}(B)$ by the 
formula

$$
k(\Phi)_{x}\left(v_{1} \wedge \ldots \wedge v_{s}\right)=\int_{0}^{1}\left(\bar{H}^{*} \Phi\right)_{(t, x)}\left(\left.\frac{\partial}{\partial t}\right|_{(t, x)} \wedge v_{1} \wedge \ldots \wedge v_{s}\right) d t
$$

which implies that $H$ and $H^{\prime}$ induce the same mappings on cohomology.

If $\left(A, \llbracket \cdot, \cdot \rrbracket, \omega_{A}\right)$ and $\left(A^{\prime}, \llbracket \cdot, \cdot \rrbracket^{\prime}, \omega_{A^{\prime}}\right)$ are regular Lie algebroids over manifolds $B$ and $B^{\prime}$, respectively, and $H: A \rightarrow A^{\prime}$ is a homomorphism of these algebroids over $f: B \rightarrow B^{\prime}$, then [K4] the induced homomorphism of Lie algebra bundles $H^{+}: \boldsymbol{g}_{A} \rightarrow \boldsymbol{g}_{A^{\prime}}$ induces pullbacks of cross-sections of dual bundles and their symmetric powers $H^{+*}: \operatorname{Sec} \bigvee^{k} \boldsymbol{g}_{A^{\prime}}^{*} \rightarrow$ Sec $\bigvee^{k} \boldsymbol{g}_{A}^{*}$ given by the evident formula. The pull-back of an invariant section is again an invariant section. Hence we obtain a homomorphism of algebras $H^{+*}: I\left(A^{\prime}\right) \rightarrow I(A)$ [K4]. Assume that $L$ and $L^{\prime}$ are Lie algebroids on $B$ and $B^{\prime}$, respectively. First we check the functoriality of the homomorphism $h_{\nabla}$.

Theorem 3.3. If $\nabla: L \rightarrow A$ and $\nabla^{\prime}: L^{\prime} \rightarrow A^{\prime}$ are connections, $H_{L}: L \rightarrow L^{\prime}$ and $H_{A}: A \rightarrow A^{\prime}$ are homomorphisms of algebroids such that the diagram

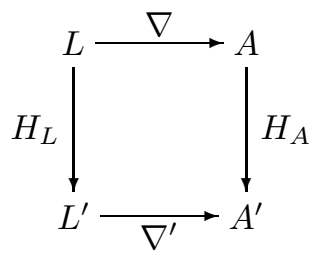

commutes, then

$$
h_{\nabla} \circ H_{A}^{+*}=H_{L}^{\#} \circ h_{\nabla^{\prime}} .
$$

Proof. Observe that

$$
\Omega_{\nabla^{\prime}}\left(H_{L}(v), H_{L}(w)\right)=H_{A}^{+}\left(\Omega_{\nabla}(v, w)\right), v, w \in L_{\mid x}, x \in B .
$$

Indeed, let $\xi, \eta$ be two cross-sections of $L$ such that $\xi(x)=v$ and $\eta(x)=w$, and consider their (local) $H_{L}$-decompositions $H_{L} \circ \xi=\sum_{i} f^{i} \cdot\left(\xi_{i}^{\prime} \circ f\right)$ and $H_{L} \circ \eta=\sum_{i} g^{i} \cdot\left(\xi_{i}^{\prime} \circ f\right)$, $\xi_{i}^{\prime} \in \operatorname{Sec} A^{\prime}$. The equality (3.4) is an immediate consequence of the fact that the diagram below is commutative

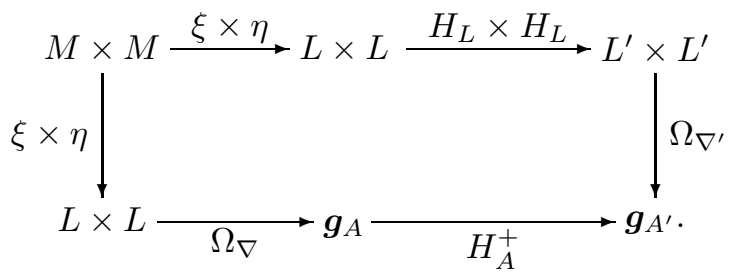

To show this, take $H_{A}$-decompositions of $\nabla \circ \xi$ and $\nabla \circ \eta, H_{A} \circ(\nabla \circ \xi)=\nabla^{\prime} \circ H_{L} \circ \xi=$ $\sum_{i} f^{i} \cdot\left(\nabla^{\prime} \circ \xi_{i}^{\prime} \circ f\right)$ (similarly for $\left.\eta\right)$. Hence

$$
\begin{aligned}
H_{A}^{+} \circ \Omega_{\nabla}(\xi, \eta) & =H_{A} \circ \llbracket \nabla \circ \xi, \nabla \circ \eta \rrbracket-H_{A} \circ \nabla \llbracket \xi, \eta \rrbracket \\
& =H_{A} \circ \llbracket \nabla \circ \xi, \nabla \circ \eta \rrbracket-\nabla^{\prime} \circ H_{L} \circ \llbracket \xi, \eta \rrbracket \\
& =\sum_{i, j} f^{i} \cdot g^{j} \cdot \llbracket \nabla^{\prime} \circ \xi_{i}^{\prime}, \nabla^{\prime} \circ \xi_{j}^{\prime} \rrbracket+\sum_{j} \omega_{A} \circ(\nabla \circ \xi)\left(g^{j}\right) \cdot \nabla^{\prime} \circ \xi_{j}^{\prime} \circ f \\
& -\sum_{i} \omega_{A} \circ(\nabla \circ \eta)\left(f^{i}\right) \cdot \nabla^{\prime} \circ \xi_{i}^{\prime} \circ f-\sum_{i, j} f^{i} \cdot g^{j} \cdot \nabla^{\prime} \circ \llbracket \xi_{i}^{\prime}, \xi_{j}^{\prime} \rrbracket \circ f
\end{aligned}
$$




$$
\begin{aligned}
& -\sum_{j}\left(\omega_{L} \circ \xi\right)\left(g^{j}\right) \cdot \nabla^{\prime} \circ \xi_{j}^{\prime} \circ f+\sum_{i}\left(\omega_{L} \circ \eta\right)\left(f^{i}\right) \cdot \nabla^{\prime} \circ \xi_{i}^{\prime} \circ f \\
& =\sum_{i, j} f^{i} \cdot g^{j} \cdot\left(\llbracket \nabla^{\prime} \circ \xi_{i}^{\prime}, \nabla^{\prime} \circ \xi_{j}^{\prime} \rrbracket-\nabla^{\prime} \circ \llbracket \xi_{i}^{\prime}, \xi_{j}^{\prime} \rrbracket\right) \circ f \\
& =\Omega_{\nabla^{\prime}} \circ\left(H_{L} \circ \xi, H_{L} \circ \eta\right)=\Omega_{\nabla^{\prime}} \circ\left(H_{L} \times H_{L}\right)(\xi, \eta) .
\end{aligned}
$$

Observe that it suffices to show that (3.3) is satisfied by forms. Let $\Gamma \in \operatorname{Sec} \bigvee^{k} \boldsymbol{g}_{A^{\prime}}^{*}, x \in$ $B$ and $v_{1}, \ldots, v_{2 k} \in L_{\mid x}$. Then

$$
\begin{aligned}
\left(\chi_{\nabla} \circ H_{A}^{+*}\right)(\Gamma)\left(x ; v_{1}, \ldots, v_{2 k}\right) & \\
= & \frac{1}{k !} \cdot\left\langle H_{A}^{+*}(\Gamma), \Omega_{\nabla} \vee \ldots \vee \Omega_{\nabla}\right\rangle\left(x ; v_{1}, \ldots, v_{2 k}\right) \\
& =\frac{1}{k !} \cdot\left\langle\Gamma_{\mid f x}, \frac{1}{2^{k}} \sum_{\sigma} \operatorname{sgn} \sigma \cdot H_{A}^{+} \circ \Omega_{\nabla}\left(x ; v_{\sigma_{1}}, v_{\sigma_{2}}\right) \vee \ldots \vee H_{A}^{+} \circ \Omega_{\nabla}\left(x ; v_{\sigma_{2 k-1}}, v_{\sigma_{2 k}}\right)\right\rangle \\
& =\frac{1}{k !} \cdot\left\langle\Gamma_{\mid f x}, \frac{1}{2^{k}} \sum_{\sigma} \operatorname{sgn} \sigma \cdot \Omega_{\nabla^{\prime}}\left(f(x) ; H_{L}\left(v_{\sigma_{1}}\right), H_{L}\left(v_{\sigma_{2}}\right)\right) \vee \ldots\right. \\
& \left.\quad \ldots \vee \Omega_{\nabla^{\prime}}\left(f(x) ; H_{L}\left(v_{\sigma_{2 k-1}}\right), H_{L}\left(v_{\sigma_{2 k}}\right)\right)\right\rangle \\
= & \frac{1}{k !} \cdot\left\langle\Gamma, \Omega_{\nabla^{\prime}} \vee \ldots \vee \Omega_{\nabla^{\prime}}\right\rangle\left(f(x) ; H_{L}\left(v_{1}\right), \ldots, H_{L}\left(v_{2 k}\right)\right) \\
= & \chi \nabla^{\prime}(\Gamma)\left(f(x) ; H_{L}\left(v_{1}\right), \ldots, H_{L}\left(v_{2 k}\right)\right) \\
= & H_{L}^{*}\left(\chi_{\nabla^{\prime}}(\Gamma)\right)\left(x ; v_{1}, \ldots, v_{2 k}\right) .
\end{aligned}
$$

THEOREM 3.4. Let $L$ and $A$ be Lie algebroids and assume that $A$ is regular. If $\nabla_{0}, \nabla_{1}$ : $L \rightarrow A$ are L-connections, then $h_{\nabla_{0}}=h_{\nabla_{1}}$.

Proof. Define $\nabla: T \mathbb{R} \times L \rightarrow T \mathbb{R} \times A$ by

$$
\nabla\left(v_{t}, w\right)=\left(v_{t},(1-t) \nabla_{0}(w)-t \nabla_{1}(w)\right), \quad v_{t} \in T_{t} \mathbb{R}, w \in L .
$$

Observe that $\nabla$ is a $T \mathbb{R} \times L$-connection, i.e. $\left(\mathrm{id}, \omega_{A}\right) \circ \nabla\left(v_{t}, w\right)=\left(\mathrm{id}, \omega_{L}\right)\left(v_{t}, w\right)$. Define now $H_{L, t}: L \rightarrow T \mathbb{R} \times L, H_{A, t}: A \rightarrow T \mathbb{R} \times A$ by $H_{L, t}(w)=\left(\theta_{t}, w\right), H_{A, t}(v)=\left(\theta_{t}, v\right)$, $w \in L, v \in A$ and $G: T \mathbb{R} \times A \rightarrow A$ by $G(v, w)=w, v \in T \mathbb{R}, w \in A$. The mappings above are non-strong homomorphisms of Lie algebroids [K4] such that $H_{A, t} \circ \nabla_{i}=\nabla \circ H_{L, t}$, $G \circ H_{A, i}=\mathrm{id}, i=0,1$. Hence by Theorem 3.3 we have

$$
\begin{aligned}
h_{\nabla_{0}} & =h_{\nabla_{0}} \circ\left(H_{A, 0}^{+*} \circ G^{+*}\right)=\left(h_{\nabla_{0}} \circ H_{A, 0}^{+*}\right) \circ G^{+*} \\
& =\left(H_{L, 0}^{\sharp} \circ h_{\nabla}\right) \circ G^{+*}=\left(H_{L, 1}^{\sharp} \circ h_{\nabla}\right) \circ G^{+*} \\
& =\left(h_{\nabla_{1}} \circ H_{A, 1}^{+*}\right) \circ G^{+*}=h_{\nabla_{1}} \circ\left(H_{A, 1}^{+*} \circ G^{+*}\right)=h_{\nabla_{1}} .
\end{aligned}
$$

According to this theorem, the Chern-Weil homomorphism defined in terms of a given $L$-connection does not depend on the choice of the connection. Thus, it will be denoted simply by $h_{L, A}$. The image of $h_{L, A}, \operatorname{Im} h_{L, A} \subset H_{L}(B)$, is a subalgebra of $H_{L}(B)$; it is called the Pontryagin algebra of $(L, A)$ and is denoted by

$$
\operatorname{Pont}(L, A) \text {. }
$$

Cohomology classes of $\operatorname{Pont}(L, A)$ are called Pontryagin classes of the pair $(L, A)$. 
Let $A$ and $L$ be Lie algebroids over a foliated manifold $(B, F)$ and consider the splitting $\mathbf{e}_{L, A}$ given by their Whitney product $A \boxplus L$. Clearly $h_{L, A}=h_{\mathbf{e}_{L, A}}$; therefore the Chern-Weil homomorphism of a pair of Lie algebroids can be obtained by using J. Huebschmann's theory. However, constructions and proofs given in our paper are simpler, and in fact, they are adapted from the case of one regular Lie algebroid, see [K4].

In general, there is no relation between $h_{L, A}$ and $h_{A, L}$ : one of them can be zero and the other one not. For example, if $A$ is a regular Lie algebroid over $(B, F)$, then $h_{A, L}=0$ and $h_{L, A}$ is not always zero.

\subsubsection{G-equivariant Chern-Weil homomorphisms}

Definition 3.5 [K6]. Let $A$ be a Lie algebroid on a manifold $B$ and let $G$ be a Lie group. $A$ is called a Lie $G$-algebroid if there is a right action of $G$ on the manifold $A$, $\widetilde{R}: A \times G \rightarrow A$, and a right action of $G$ on the manifold $B, R: B \times G \rightarrow B$, such that $\widetilde{R}_{g}: A \rightarrow A$ is an automorphism of $A$ over $R_{g}: B \rightarrow B$ for each $g \in G$.

According to this definition, the diagram

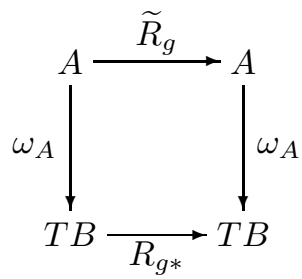

is commutative and $\widetilde{R}_{g} \circ \llbracket \xi, \eta \rrbracket=\llbracket \bar{\xi}, \bar{\eta} \rrbracket \circ R_{g}$, where $\bar{\xi}, \bar{\eta} \in \operatorname{Sec} A$ are sections such that $\widetilde{R}_{g} \circ \xi=\bar{\xi} \circ R_{g}$ and $\widetilde{R} \circ \eta=\bar{\eta} \circ R_{g}$. This definition of a Lie $G$-algebroid is more general than the definition of a Mackenzie $P B G$-algebroid [M4] (recall that a transitive $G$-algebroid $A$ is a $P B G$-algebroid if the base manifold is the total space of a principal bundle $P(B, G, p)$ and the action of $G$ on $P$ is the structural action of this bundle).

Assume that $L$ and $A$ are Lie $G$-algebroids on a base $B$.

Definition 3.6. An $L$-connection $\chi$ in $A, \chi: L \rightarrow A$, is called $G$-equivariant if $\chi$ is a $G$-equivariant homomorphism.

Assume additionally that $A$ is regular and $0 \rightarrow \boldsymbol{g}_{A} \rightarrow A \rightarrow F \rightarrow 0$ is its Atiyah sequence. Clearly, the bundle $\boldsymbol{g}_{A}$ is $G$-invariant. Let $\Omega: L \times L \rightarrow \boldsymbol{g}_{A}$ be the curvature form of a $G$-equivariant $L$-connection $\chi$ in $A$. It is standard to check that

$$
\Omega\left(R_{g} v, R_{g} w\right)=R_{g} \Omega(v, w)
$$

which means that the $2 k$-form $\langle\Gamma, \Omega \vee \ldots \vee \Omega\rangle$ is $G$-equivariant for a $G$-equivariant section $\Gamma \in \operatorname{Sec} \bigvee^{k} \boldsymbol{g}_{A}^{*}$. Hence we obtain the $G$-equivariant Chern-Weil homomorphism for the pair $(L, A)$

$$
h_{L, A}^{G}: I(A)^{G} \rightarrow H_{L}^{G}(B)
$$

where the upper index $G$ denotes the space of $G$-equivariant elements of $I(A)$ and the cohomology of $G$-equivariant forms respectively. The proof that $h_{L, A}^{G}$ does not depend on the choice of the $G$-equivariant connection is nearly the same as the proof of Theorem 3.4. The difference is that we introduce the Lie $G$-algebroids $T \mathbb{R} \times L$ and $T \mathbb{R} \times A$ given by $R_{g}\left(v_{t}, w\right)=\left(v_{t}, R_{g} w\right)$, and next we observe that the joining connection is $G$-equivariant 
and the homomorphisms $H_{L, t}, H_{A, t}$ and $T$ are $G$-equivariant as well. Moreover, $H_{L, 0}$ and $H_{L, 1}$ are homotopic via a $G$-equivariant homotopy.

Similarly, if we consider an extension of Lie algebroids

$$
\mathbf{e}: 0 \rightarrow K \rightarrow A \stackrel{\pi}{\rightarrow} L \rightarrow 0,
$$

where $A$ and $L$ are Lie $G$-algebroids and $\pi$ is a $G$-equivariant homomorphism, then we obtain the $G$-equivariant Chern-Weil homomorphism for the extension e in terms of $G$-equivariant connections

$$
h_{\mathbf{e}}^{G}: I(K)^{G} \rightarrow H_{L}^{G}(B) .
$$

3.2.4. Comparison of $h_{L, A}$ with $h_{A}$

THEOREM 3.7. If $L$ and $A$ are Lie algebroids over a manifold $B, A$ is regular over $(B, F)$ and $\operatorname{Im} \omega_{L} \subset F$, then the diagram

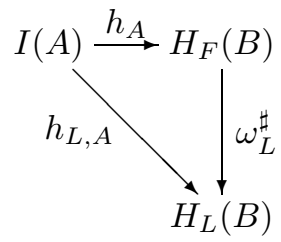

commutes. In particular

$$
\omega_{L}^{\sharp}[\operatorname{Pont}(A)]=\operatorname{Pont}(L, A) .
$$

Proof. Consider the $L$-connection $\nabla: L \rightarrow A$ given by $\nabla=\lambda \circ \omega_{L}$, where $\lambda: F \rightarrow A$ is a connection in $A$. It is easy to show that the curvature tensors $\Omega_{\lambda} \in \Omega_{F}^{2}\left(B, \boldsymbol{g}_{A}\right)$ and $\Omega_{\nabla} \in \Omega_{L}^{2}\left(B, \boldsymbol{g}_{A}\right)$ satisfy

$$
\Omega_{\nabla}=\omega_{L}^{*}\left(\Omega_{\lambda}\right)
$$

Hence for an invariant section $\Gamma$ of the symmetric polynomials bundle we obtain

$$
\omega_{L}^{*} \circ \chi_{\lambda}(\Gamma)=\frac{1}{k !}\left\langle\Gamma, \omega_{L}^{*} \Omega_{\lambda} \vee \ldots \vee \omega_{L}^{*} \Omega_{\lambda}\right\rangle=\chi_{\nabla}(\Gamma)
$$

An immediate result is the implication

$$
h_{A}=0 \text { ongrightarrowh } h_{L, A}=0,
$$

for each pair $(L, A)$ of Lie algebroids over a foliated manifold $(B, F)$. Hence, if $A$ is a Lie algebroid with Atiyah sequence $0 \rightarrow g_{A} \rightarrow A \rightarrow F \rightarrow 0$ and if we consider an $L$-connection in $A$ for another Lie algebroid $L$, then it can turn out that the amount of obstructions for the existence of a flat $L$-connection will decrease. In the special case $L=A$ we obtain no such obstructions: $h_{A, A}=0$ since $\operatorname{id}_{A}: A \rightarrow A$ is a flat $A$-connection in $A$. Hence the diagram below is commutative

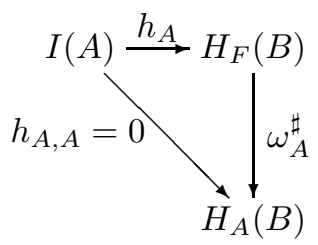

which means that

$$
\operatorname{Pont}(A) \subset \operatorname{ker} \omega_{A}^{\sharp},
$$


i.e. the images of Pontryagin classes under the anchor $\omega_{A}$ are zero: for an invariant section $\Gamma \in I(A)$ of rank $k$ and any connection $\chi$ in $A$

$$
\omega_{A}^{*}\left(\left\langle\Gamma, \Omega_{\lambda} \vee \ldots \vee \Omega_{\lambda}\right\rangle\right)=d \Phi,
$$

for some form $\Phi$. One of that forms is given in [K10]; let $\eta: A \rightarrow \boldsymbol{g}_{A}$ be the form of $\lambda$. Then

$$
\Phi=k ! \sum_{i+j=k-1} \frac{1}{k+j} \cdot\left\langle\Gamma, \eta \vee \frac{1}{i !}\left(d_{A} \eta\right)^{i} \vee \frac{1}{j !}\left(-\frac{1}{2} \llbracket \eta, \eta \rrbracket\right)^{j}\right\rangle .
$$

Corollary 3.8. If $\omega_{L}^{\sharp}: H_{F}(B) \rightarrow H_{L}(B)$ is a monomorphism, then

$$
h_{A}=0 \Longleftrightarrow h_{L, A}=0 \text {. }
$$

In the class of transitive unimodular invariantly oriented Lie algebroids $L$ on an oriented manifold $B[\mathrm{~K} 8], \omega_{L}^{\sharp}$ is a monomorphism if and only if the fibre integral of forms with fibre-compact carrier $f_{L, c}^{\sharp}: H_{L, c}(B) \rightarrow H_{c}(B)[\mathrm{K} 9]$ is an epimorphism. In the smaller class of so-called $s$-Lie algebroids [K10], $\omega_{L}^{\sharp}$ is a monomorphism if and only if the Euler class of a Lie algebroid $L, \chi_{E}$, vanishes (for example, if $H^{n+1}(B)=0$, where $\left.n=\operatorname{rank} \boldsymbol{g}_{L}\right)$.

3.2.5. Comparison of $h_{L, A}$ with Fernandes L-Chern-Weil homomorphism. Fernandes [F2] has constructed an $L$-Chern-Weil homomorphism $h_{L, P}: I(G) \rightarrow H_{L}(B)$ of a $G$ principal fibre bundle $P(B, G)$ in such a way that $h_{L, P}=\omega_{L}^{\sharp} \circ h_{P}$ (see Proposition 4.3 in [F2]). According to the diagrams (1.2) and (3.5) the Chern-Weil homomorphisms $h_{L, A(P)}$ and $h_{L, P}$ are joined via the following diagram

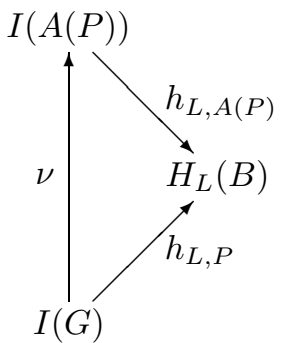

If $P$ is connected then identifying $I(G)=I(A(P))$ we obtain $h_{L, P}=h_{L, A(P)}$ which proves the algebroid nature of the Fernandes $L$-Chern-Weil homomorphism.

3.2.6. Comparison of $h_{L, A}$ with the Chern-Weil homomorphism of an extension. Let $A$ and $L$ be two Lie algebroids on a manifold $B$ and let $\pi: A \rightarrow L$ be a given epimorphism of Lie algebroids. Then $L^{\prime}:=\operatorname{ker} \pi$ is a subbundle of $A$.

LEMMA 3.9. There is precisely one structure of a Lie algebroid in $L^{\prime}$ for which

$$
\mathbf{e}(\pi): 0 \rightarrow L^{\prime} \stackrel{i}{\rightarrow} A \stackrel{\pi}{\rightarrow} L \rightarrow 0
$$

is a short exact sequence of Lie algebroids. The anchor of $L^{\prime}$ is $\omega_{L^{\prime}}=0$.

Proof. The uniqueness is a consequence of the fact that the anchor and Lie bracket must be given by $\omega_{L^{\prime}}=\omega_{A} \circ i$ and $\llbracket \xi, \eta \rrbracket^{L^{\prime}}=\llbracket \xi, \eta \rrbracket^{A}$ for $\xi, \eta \in \operatorname{Sec} L^{\prime}$. Observe now that

$$
\omega_{L^{\prime}}\left(\xi^{\prime}\right)=\omega_{A} \circ i\left(\xi^{\prime}\right)=\left(\omega_{L} \circ \pi\right) i\left(\xi^{\prime}\right)=\omega_{L} \circ(\pi \circ i)\left(\xi^{\prime}\right)=0 .
$$


Given an epimorphism $\pi: A \rightarrow L$ and the induced extension $\mathbf{e}(\pi)$ consider the structural diagram:

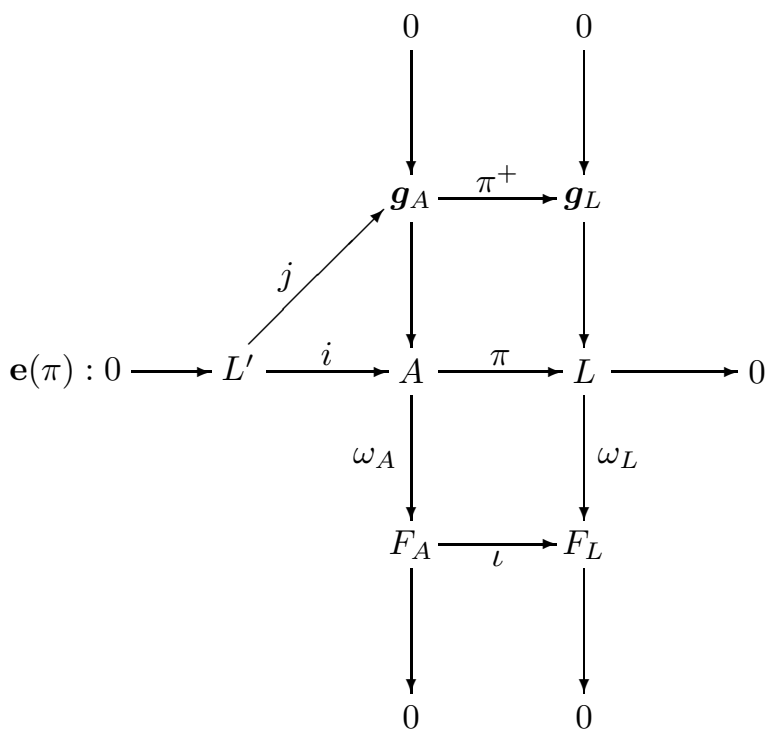

The existence of the $\mathbf{e}(\pi)$-connection implies the equality $F_{A}=F_{L}$.

There is a representation $\operatorname{ad}_{\mathbf{e}(\pi)}$ of the Lie algebroid $A$ in the bundle $L^{\prime}$ satisfying $\operatorname{ad}_{\mathbf{e}(\pi)}(\xi)\left(\eta^{\prime}\right)=\llbracket \xi, \eta^{\prime} \rrbracket^{A}, \eta^{\prime} \in \operatorname{Sec} L^{\prime}, \xi \in \operatorname{Sec} A$. Indeed, $\llbracket \xi, \eta^{\prime} \rrbracket^{A} \in \operatorname{Sec} L^{\prime}$ and $\operatorname{ad} \mathbf{e}_{\mathbf{e}(\pi)}$ : $\operatorname{Sec} L^{\prime} \rightarrow \operatorname{Sec} L^{\prime}$ is a covariant differential operator with the anchor $\omega_{A}(\xi) \cdot \operatorname{ad} d_{\mathbf{e}(\pi)}$ and the adjoint representation $\operatorname{ad}_{A}$ of $A$ induces representations in $\bigvee^{k} L^{\prime}$ and $\bigvee^{k} \boldsymbol{g}_{A}^{*}, k=1,2, \ldots$ given by (2.15) where the domains are the algebras of invariant sections $I(\mathbf{e})$ and $I(A)$, respectively. If $\Gamma \in \operatorname{Sec} \bigvee^{k} \boldsymbol{g}_{A}^{*}$ is $\operatorname{ad}_{A^{-}}$-invariant, then the restriction $\Gamma_{\mid L^{\prime}} \in \operatorname{Sec} \bigvee^{k} L^{\prime *}$ is $\operatorname{ad}_{\mathbf{e}(\pi)}$-invariant and

$$
I(A) \rightarrow I(\mathbf{e}), \quad \Gamma \mapsto \Gamma_{\mid L^{\prime}},
$$

is a homomorphism of algebras. Characteristic classes from the image of the Chern-Weil homomorphims of an extension $\mathbf{e}(\pi)$ of Lie-Rinehart algebras (see [H2] and [T2]) give obstructions to the existence of a flat $\mathbf{e}(\pi)$-connection. In brief, we give the construction of the Chern-Weil homomorphism of an extension $\mathbf{e}(\pi)$. The curvature tensor of an $\mathbf{e}(\pi)$ connection $\nabla: L \rightarrow A$ is a 2 -form $\Omega_{\nabla}^{\mathbf{e}(\pi)} \in \Omega_{L}^{2}\left(B ; L^{\prime}\right)$ such that

$$
\Omega_{\nabla}^{\mathbf{e}(\pi)}=\llbracket \nabla \xi, \nabla \eta \rrbracket^{A}-\nabla \llbracket \xi, \eta \rrbracket^{L} .
$$

Since $\nabla$ is an $L$-connection in $A$ as well, it possesses its curvature tensor $\Omega_{\nabla} \in \Omega_{L}^{2}\left(B ; \boldsymbol{g}_{A}\right)$ which is given by (3.6). Hence

$$
\Omega_{\nabla}=j \Omega_{\nabla}^{\mathbf{e}(\pi)}
$$

The Chern-Weil homomorphism $h_{\mathbf{e}(\pi)}$ of the extension $\mathbf{e}(\pi)$ satisfies

$$
h_{\mathbf{e}(\pi)}(\Gamma)=\frac{1}{k !} \cdot\left[\left\langle\Gamma, \Omega_{\nabla}^{\mathbf{e}(\pi)} \vee \ldots \vee \Omega_{\nabla}^{\mathbf{e}(\pi)}\right\rangle\right] .
$$


The four Chern-Weil homomorphisms $h_{A}, h_{L}, h_{L, A}, h_{\mathbf{e}(\pi)}$ ( $A$ and $L$ are regular Lie algebroids over a foliated manifold $(B ; F))$ are connected by the commutative diagram

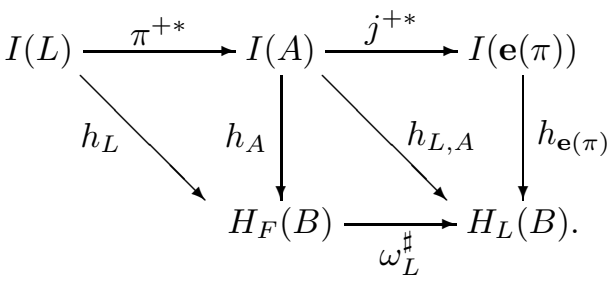

Commutativity of the left-hand side triangle is a consequence of the fact that the Chern -Weil homomorphism of regular Lie algebroids is functorial. The next triangle is commutative by Theorem 3.7. To see that the triangle on the right-hand side is commutative we compute

$$
\begin{aligned}
h_{\mathbf{e}(\pi)}\left(j^{+*}(\Gamma)\right) & =\frac{1}{k !} \cdot\left[\left\langle j^{+*} \Gamma, \Omega_{\nabla}^{\mathbf{e}(\pi)} \vee \ldots \vee \Omega_{\nabla}^{\mathbf{e}(\pi)}\right\rangle\right] \\
& =\frac{1}{k !} \cdot\left[\left\langle\Gamma, j \Omega_{\nabla}^{\mathbf{e}(\pi)} \vee \ldots \vee j \Omega_{\nabla}^{\mathbf{e}(\pi)}\right\rangle\right] \\
& =\frac{1}{k !} \cdot\left[\left\langle\Gamma, \Omega_{\nabla} \vee \ldots \vee \Omega_{\nabla}\right\rangle\right]=h_{A}(\Gamma) .
\end{aligned}
$$

$h_{L, A} \circ \pi^{+*}=0$ since $\operatorname{Im} h_{L} \subset \operatorname{ker} \omega_{L}^{\sharp}$. Hence, if $\pi^{+*}$ is an epimorphism, then $h_{L, A}=0$.

In the case of $G$-Lie algebroids we can put the superscript ${ }^{G}$ in the diagram (3.8).

Problem 3.10. Find an example of an extension $\mathbf{e}$ with $h_{\mathbf{e}} \neq 0$ (then there is no flat e-connection) and such that there is a flat $L$-connection in $A$ (in particular $h_{L, A}=0$ ). In this example $j^{+*}$ cannot be a surjective homomorphism. A wide class of objects with an epimorphism $j^{+*}$ (in the non-transitive case where $\pi: A \rightarrow L$ comes from an extension of principal bundles $Q(B, H, q) \stackrel{\pi}{\rightarrow} P(B, G, p)$ with respect to an extension of Lie groups $0 \rightarrow N \rightarrow H \rightarrow G \rightarrow 0$ ) was given by $K$. Mackenzie [M5]. It is true when $N, H$ and $G$ are compact and connected. The problem is to study the non-compact case which can be extended to the case of Lie algebroids.

3.2.7. Induced tangential case. If $A$ is a transitive Lie algebroid and $\operatorname{Im} \omega_{L}$ is contained in a regular foliation $F \subset T B$, then together with an $L$-connection $\nabla: L \rightarrow A$ we can consider an induced tangential $L$-connection $\nabla^{F}: L \rightarrow A^{F}$ defined by the same formula (see Introduction). According to Theorem 3.7 we have the following diagram joining the Chern-Weil homomorphisms $h_{L, A}$ and $h_{L, A^{F}}$.

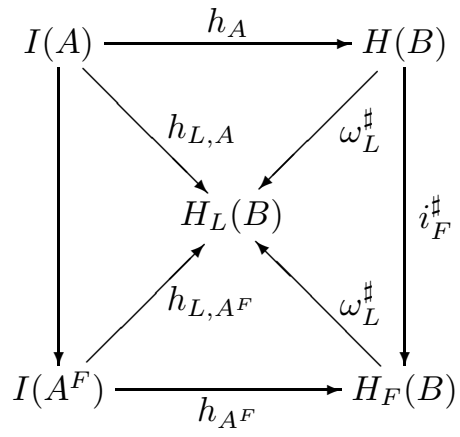


Since - in general - the domain $I\left(A^{F}\right)$ is not generated as an $\Omega_{b}(B, F)$-module by $I(A)$ (see $[\mathrm{K} 5]$ ) we can pose the problem:

Problem 3.11. Find an example in which $h_{L, A}=0$ and $h_{L, A^{F}} \neq 0$.

\section{Transversal Lie algebroids and transversal lifting of connections}

4.1. Mackenzie algebroid. Consider an extension e : $0 \rightarrow K \rightarrow A \stackrel{\pi}{\rightarrow} T P_{/ G} \rightarrow 0$ of transitive Lie algebroids where $T P_{/ G}$ is the Lie algebroid of a principal fibre bundle $P$. Recall the construction (given by K. Mackenzie) of a lifting of $\mathbf{e}$ via a projection $p: P \rightarrow B$ to some $P B G$-Lie algebroid $p^{*} A$ on $P$ with the Atiyah sequence

$$
0 \rightarrow p^{*} K \rightarrow p^{*} A \stackrel{\widetilde{q}}{\rightarrow} T P \rightarrow 0,
$$

where $\widetilde{q}$ is a lifting of $\pi$ (under the identification $T P \cong p^{*}\left(T P_{/ G}\right)$ ). We identify also $\operatorname{Sec}\left(p^{*} A\right) \cong C^{\infty}(P) \otimes_{C^{\infty}(B)} \operatorname{Sec} A$. The reader can find the next theorems in [M5].

THEOREM 4.1 (K. Mackenzie). There is a structure of a Lie algebroid in the bundle $p^{*} A$. The anchor is $\widetilde{q}$ and sections of $p^{*} A$ satisfy

$$
\llbracket f \otimes \xi, f \otimes \eta \rrbracket=f \cdot g \cdot \llbracket \xi, \eta \rrbracket+f \cdot(\pi \circ \xi) \rightarrow(g) \otimes \eta-g \cdot(\pi \circ \eta) \rightarrow(f) \otimes \xi,
$$

where $X \rightarrow$ denotes the right-invariant vector field on $P$ induced by $X \in \operatorname{Sec}\left(T P_{/ G}\right)$.

The canonical homomorphisms $\bar{p}$ and $\overline{\bar{p}}$ in the diagram

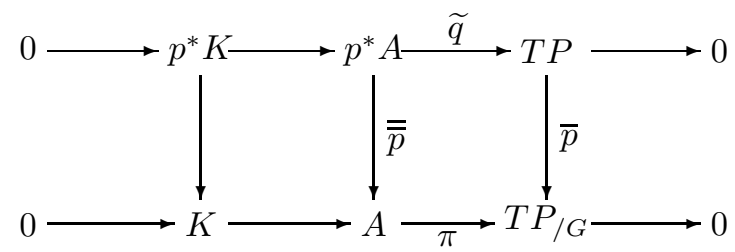

are non-strong homomorphisms of Lie algebroids (over $p: P \rightarrow B$ ). Notice that $p^{*} A$ is not a pull-back of $A$ according to the definitions in [H-M] and [K4].

Theorem 4.2 (K. Mackenzie). A right action $R$ of $G$ on $p^{*} A$ is given by

$$
R_{g}(z, v)=(z g, v) .
$$

$R_{g}: p^{*} A \rightarrow p^{*} A$ is an automorphism of the Lie algebroid $p^{*} A$ over the right translation $R_{g}: P \rightarrow P$ and hence $p^{*} A$ is a $P B G$-algebroid.

Definition 4.3. The transitive algebroid $p^{*} A$ on $P$ (constructed above) will be called the Mackenzie algebroid of an extension e or the algebroid transversal to e (by analogy with the notion a transversal principal bundle when e comes from an extension of principal bundles [M5]).

Proposition 4.4. If $\chi: T P_{/ G} \rightarrow A$ is an $\mathbf{e}$-connection, then its lifting $\tilde{\chi}=p^{*}(\chi)$ is a $G$-equivariant connection in the transversal algebroid $p^{*} A$. Conversely, any $G$-equivariant connection in $p^{*} A$ is of this form.

The last proposition corresponds to Prop. 3.2 in [M5] for extensions of principal bundles. $\widetilde{\chi}$ will be called the transversal lifting of the e-connection $\chi$. 
4.2. Some generalizations of connection. We now study lifting of any $T P_{/ G}$-connection in $A$.

Proposition 4.5. Let $\chi: T P_{/ G} \rightarrow A$ be an arbitrary $T P_{/ G}$-connection in $A$ (i.e. a linear homomorphism with $\left.\omega_{A} \circ \chi=\omega\right)$. Then, the lifting $\tilde{\chi}: T P \rightarrow p^{*} A$ is a G-equivariant homomorphism such that $p_{*}(\widetilde{q} \circ \tilde{\chi})=p_{*}$ (i.e. the difference between $\widetilde{q} \circ \tilde{\chi}(v)$ and $v$ is a vertical vector, one which is tangent to an orbit of the action of $G$ on $P)$. Conversely, if $\gamma: T P \rightarrow p^{*} A$ is a G-equivariant homomorphism with $p_{*}(\widetilde{q} \circ \tilde{\chi})=p_{*}$, then $\gamma=\tilde{\chi}$ for some $T P_{/ G^{-}}$connection $\chi$ in $A$.

Proof. "ongrightarrow" It is easy to show that $\widetilde{\chi}$ is $G$-equivariant. To see that $p_{*}(\widetilde{q} \circ$ $\tilde{\chi})=p_{*}$ consider the diagram

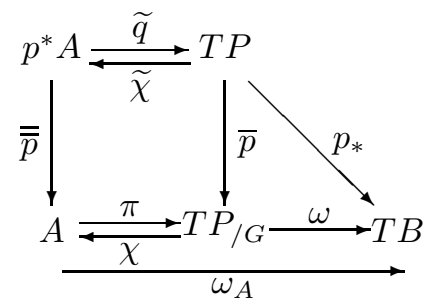

Then

$$
p_{*} \circ(\widetilde{q} \circ \tilde{\chi})=\omega_{A} \circ \overline{\bar{p}} \circ \tilde{\chi}=\omega_{A} \circ \chi \circ \bar{p}=\omega \circ \bar{p}=p_{*} .
$$

"ongleftarrow" Let $\gamma: T P \rightarrow p^{*} A$ be a $G$-equivariant linear homomorphism with $p_{*}(\widetilde{q} \circ \tilde{\chi})=p_{*}$. Since $\gamma$ is $G$-equivariant, there is a linear homomorphism of vector bundles $\chi: T P_{/ G} \rightarrow A$, and its lifting is $\gamma$. It remains to check that $\omega_{A} \circ \chi=\omega$. Since $\bar{p}$ is a linear homomorphism on the fibres, we have

$$
\omega_{A} \circ \chi \circ \bar{p}=\omega_{A} \circ \overline{\bar{p}} \circ \gamma=p_{*} \circ \widetilde{q} \circ \gamma=p_{*}=\omega \circ \bar{p} .
$$

The proposition above suggests that we should generalize the notion of a connection in a regular $G$-Lie algebroid $A \stackrel{\omega_{A}}{\rightarrow} F$ on $B$ as follows: a linear homomorphism $\lambda: F \rightarrow A$ is called a $G$-connection if the difference between $\omega_{A} \circ \lambda(v)$ and $v, v \in F$, is a vector tangent to an orbit of the action $G$ on $B$ (if the group $G$ is trivial, then we obtain the standard connection in $\left.A, \omega_{A} \circ \lambda=\mathrm{id}\right)$. Under this definition we can say that the transversal lifting

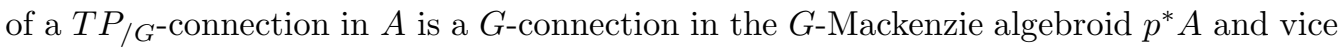
versa.

4.3. Geometric interpretation of a Mackenzie algebroid. Recall the geometric interpretation of a Mackenzie algebroid [M5]. Let (2.5) be an extension of principal bundles (with respect to an extension of Lie groups $0 \rightarrow N \rightarrow H \stackrel{\pi}{\rightarrow} G \rightarrow 0$ ) and consider the corresponding exact sequence (an extension of transitive Lie algebroids)

$$
\mathbf{e}: 0 \rightarrow Q \times_{H} \mathfrak{n} \rightarrow T Q_{/ H} \stackrel{\pi_{*}^{G}}{\rightarrow} T P_{/ G} \rightarrow 0 .
$$

Let $Q(P, N, \pi)$ be an $N$-principal bundle transversal to the extension of principal bundles and let $T Q_{/ N}$ be its Lie algebroid with the Atiyah sequence

$$
0 \rightarrow Q \times_{N} \mathfrak{n} \rightarrow T Q / N \stackrel{\pi_{*}^{N}}{\rightarrow} T P \rightarrow 0 .
$$


The Lie group $G$ acts on $T Q_{/ N}$ by

$$
\widetilde{R}_{g}: T Q_{/ N} \rightarrow T Q_{/ N}, \quad\left[X_{v}\right]^{N} \mapsto\left[R_{h *} X_{v}\right]^{N},
$$

where $X_{v} \in T_{v} Q$ and $h \in \pi^{-1}(g) . \widetilde{R}_{g}$ is an automorphism of the Lie algebroid $T Q_{/ N}$ over the right translation $R_{g}: P \rightarrow P$. Under this action, $T Q_{/ N}$ is a $P B G$-Lie algebroid. The homomorphism $\widetilde{R}_{g}: T Q_{/ N} \rightarrow T Q_{/ N}$ has a (not necesarily unique) lifting to an automorphism of the $N$-principal bundle $\pi: Q \rightarrow Q, z \mapsto z h$, with respect to an automorphism of Lie group $\tau_{h^{-1}}: N \rightarrow N, n \mapsto h^{-1} n h$. Given $g \in G$ denote by $\alpha(g)$ any element from $\pi^{-1}(g)$. The family of these automorphisms gives a smooth mapping $Q \times G \rightarrow Q$ if and only if $\alpha$ is a smooth mapping, which is equivalent to the fact that the quotient bundle $H \rightarrow H_{/ N}=G$ is trivial. Hence there is usually no action of the Lie group $G$ on the transversal bundle $Q$ which gives $\widetilde{R}$.

Situation like this seems to be more elegant from the Lie groupoids point of view: given the Lie-Ehresmann groupoid $T=Q \times Q_{/ N}$ of a transversal bundle $Q(P, N, \pi)$ there is a right action (Mackenzie) $R: T \times G \rightarrow T$ given by $R_{g}\left(<v_{2}, v_{1}>\right)=<v_{2} h, v_{1} h>, h \in \pi^{-1}(g)$. The differential of $R_{g}$ defines an automorphism of the Lie algebroid $A(T)$ of $T$ and under the canonical identification $A(T) \cong T Q_{/ N}$ we obtain an action $\tilde{R}$ which is given as above. The advantage of the groupoid and algebroid approach in comparison with principal bundles is now clear (see [M5, Remark (1) ]).

A connection $\chi: T P_{/ G} \rightarrow T Q_{/ H}$ in the extenision e has a unique lifting to a connection $\tilde{\chi}: T P \rightarrow T Q_{/ N}$ in the algebroid $T Q_{/ N}$ (and next to a connection in the transversal bundle). Indeed, if $\chi\left(\left[u_{z}\right]^{G}\right)=\left[X_{v}\right]^{H}\left(X_{v} \in T_{v} Q, \pi z=h\right)$, then $\widetilde{\chi}\left(u_{z}\right)=\left[X_{v}\right]^{N}$. Observe that $\widetilde{R}_{g}$ induces an automorphism of $p^{*}\left(T Q_{/ H}\right),(z, \alpha) \mapsto(z g, \alpha)$. The connection $\tilde{\chi}$, we have obtained is $G$-equivariant and conversely, each $G$-equivariant connection $\gamma: T P \rightarrow T Q_{/ N}$ has the form of $\tilde{\chi}$ for some connection $\chi$ in e. Under the identification $T Q_{/ N} \cong p^{*}\left(T Q_{/ H}\right)$, we can introduce the Lie algebroid structure in $p^{*}\left(T Q_{/ H}\right)$ obtaining a Mackenzie algebroid.

4.4. The equivariant Chern-Weil homomorphism of a Mackenzie algebroid. Consider an extension of Lie algebroids e : $0 \rightarrow K \rightarrow A \rightarrow T P_{/ G} \rightarrow 0$ and the corresponding transversal $P B G$-Mackenzie algebroid $0 \rightarrow p^{*} K \rightarrow p^{*} A \rightarrow T P \rightarrow 0$. According to Section 3.2.3, we have the well defined $G$-equivariant Chern-Weil homomorphism $h_{p^{*} A}^{G}: I\left(p^{*} A\right)^{G} \rightarrow H^{G}(P)$ and this homomorphism is equivalent to the Chern-Weil homomorphism of the extension e under the canonical isomorphisms

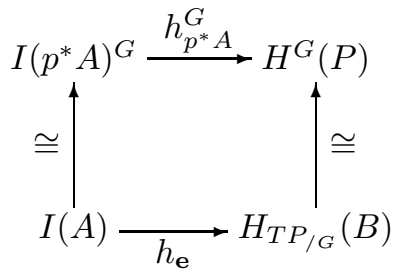

If manifolds $P$ and $Q$ in the extension $Q(B, H, q) \stackrel{\pi}{\rightarrow} P(B, G, p) \rightarrow 0$ are connected (the Lie groups $H$ and $G$ need not to be connected), then $I(G) \cong I\left(T P_{/ G}\right), I(H) \cong I\left(T Q_{/ H}\right)$, $I(N) \cong I\left(T Q_{/ N}\right)$ and in consequence, $I(N)^{G} \cong I\left(T Q_{/ N}\right)^{G}$. Hence, given an extension 
e which comes from an extension of principal bundles $Q \rightarrow P$, the diagram (3.8) (if we omit $h_{L, A}$ ) is equivalent to the diagram (1.3) given by K. Mackenzie.

Acknowledgments. The authors are grateful to Prof. Izu Vaisman for suggestions and opinions which were very helpful during their work on the construction of the characteristic homomorphism for a pair of Lie algebroids.

\section{References}

[B1] I. W. Belko, Characteristic classes of transitive Lie algebroids, preprint Minsk, 1994, 192 pp. (in Russian).

[B2] I. W. Belko, Characteristic homomorphism of a Lie algebroid, News Belarus Acad. Sci. Ser. phys.-math. sci. 1, 1997 (in Russian).

[C] M. Crainic, Differentiable and algebroid cohomology, Van Est isomorphisms, and characteristic classes, Preprint DG/0008064.

[F1] R. L. Fernandes, Connections in Poisson geometry I: holonomy and invariants, J. Differential Geometry 54 (2000), 303-366 (math.DG/0001129).

[F2] R. L. Fernandes, Lie algebroids, holonomy and characteristic classes, preprint DG/007132.

[G] W. Greub, Multilinear Algebra, Springer, 1967.

[G-H-V] W. Grueb, S. Halperin and R. Vanstone, Connections, Curvature and Cohomology, Vols. I, II, Academic Press, 1972, 1973.

[H] J.-C. Herz, Pseudo-algèbres de Lie, C. R. Acad. Sci. Paris 236 (1953), I, 1935-1937 and II, 2289-2291.

[H-M] P. J. Higgins and K. Mackenzie, Algebraic constructions in the category of Lie algebroids, J. Algebra (1988).

[H1] J Huebschmann, Poisson cohomology and quantization, J. Reine Angew. Math. 408 (1990), 57-113,

[H2] - Extensions of Lie-Rinehart algebras and the Chern-Weil homomorphism, in: Contemp. Math. 227, 1999.

[IKV] V. Itskov, M. Karasev and Y. Vorobjev, Infinitesimal Poisson cohomology, in: AMS Transl. (2) 187, 1998.

[K-T] F. Kamber and P. Tondeur, Foliated Bundles and Characteristic Classes, Lectures Notes in Math. 493, Springer, 1975.

[K] J. L. Koszul, Lectures on Fibre Bundles and Differential Geometry, Tata Institute of Fundamental Research, Bombay, 1960, reprinted by Springer, 1986.

[K1] J. Kubarski, Exponential mapping for Lie groupoids. Applications, Colloq. Math. 54 (1987), 39-48.

[K2] - Pradines-type groupoids over foliations; cohomology, connections and the ChernWeil homomorphism, Technical University, Institute of Mathematics, Preprint 2, August 1986.

[K3] -, Lie algebroid of a principal fibre bundle, Publ. Dép. Math. Univ. Lyon 1, 1/A, 1989.

[K4] - The Chern-Weil homomorphism of regular Lie algebroids, Publ. Dép. Math. Univ. Lyon 1, 1991.

[K5] - Tangential Chern-Weil homomorphism, in: Geometric Study of Foliations (Tokyo, 1993), ed. by T. Mizutani, World Sci., 1994, 327-344,

[K6] - Invariant cohomology of regular Lie algebroids, in: Analysis and Geometry in Foliated Manifolds (Santiago de Compostela, 1994), World Sci., 1995. 
[K7] -, Bott's vanishing theorem for regular Lie algebroids, Trans. A.M.S. 348 (1996).

[K8] - Fibre integral in regular Lie algebroids, in: New Developments in Differential Geometry (Budapest, 1996), Kluwer, 1999, 173-202.

[K9] - Poincaré duality for transitive unimodular invariantly oriented Lie algebroids, Topology Appl., to appear.

[K10] -, Gysin sequence and Euler class of spherical Lie algebroids, Publ. Math. Debrecen (in print).

[K-W] J. Kubarski and W. Walas, Mackenzie algebroid, Poster 514, ECM 2000.

[L] P. Libermann, Pseudogroupes infinitésimaux attachés aux pseudogroupes de Lie, Bull. Soc. Math. France 87 (1959), 409-425,

[Lis] K. Lisiecki, On the generating of a topological groupoid by its open subset containing all units, Sci. Bull. Łódź Technical University, Mat., no. 23, 1993.

[M1] K. Mackenzie, Lie Groupoids and Lie Algebroids in Differential Geometry, London Math. Soc. Lecture Note Ser. 124, Cambridge, 1987.

[M2] -, On extensions of principal bundles, Ann. Global Anal. Geom. 6 (1988), 141-163,

[M3] - Lie algebroids and Lie pseudoalgebras, Bull. London Math. Soc. 27 (1995), 97-147.

[M4] - Integrability obstructions for extensions of Lie algebroids, Cahiers Topol. Géom. Diff. Catégoriques 28 (1987), 29-52.

[M5] -, On extensions of principal bundles, Ann Global Anal. Geom. 6 (1988), 141-163.

[M-C] C. Moore and C. Schochet, Global Analysis on Foliated Spaces, Math. Sci. Res. Inst. Publications 9, Springer, 1988.

[NVQ] Ngo Van Que, De la connexion d'ordre supérieur, C. R. Acad. Sci. Paris 259, 2061-2064, [MR] L. Maxim-Raileanu, Cohomology of Lie algebroids, An. Sti. Univ. "Al. I. Cuza" Iasi. Sect. I a Mat. 22 (1976), 197-199,

[P] R. Palais, Seminar on the Atiyah-Singer Index Theorem, Princeton Univ. Press, 1965.

[Pr] J. Pradines, Théorie de Lie pour les groupoïdes différentiables, Atti del Convegno Internazionale di Geometria Differenziale, Bologna, 1967.

[S] D. Spencer, Overdetermined systems of linear partial differential equations, Bull. Amer. Math. Soc. 75 (1969), 179-239,

[T1] N. Teleman, Cohomology of Lie algebras, in: Global Analysis and its Applications (Trieste, 1972), Vol. III, Intern. Course, Vienna 1974.

[T2] - A characteristic ring of a Lie algebra extension, Accad. Naz. Lincei Rend. Cl. Sci. Fis. Mat. Natur. 8 (1972), 498-506 and 708-711.

[V1] I. Vaisman, On the geometric quantization of Poisson manifolds, J. Math. Physics 32 (1991), 3339-3345.

[V2] - Lectures on the Geometry of Poisson Manifolds, Birkhäuser, 1994. 